\title{
Detecting Global Predicates in Distributed Systems with Clocks
}

\author{
Scott D. Stoller* \\ Computer Science Dept., Indiana University, Bloomington, IN 47405 USA
}

February 7, 2000

\begin{abstract}
This paper proposes a framework for detecting global state predicates in systems of processes with approximately-synchronized real-time clocks. Timestamps from these clocks are used to define two orderings on events: "definitely occurred before" and "possibly occurred before". These orderings lead naturally to definitions of 3 distinct detection modalities, i.e., 3 meanings of "predicate $\Phi$ held during a computation", namely: Poss $\stackrel{d b}{\rightarrow} \Phi$ (" $\Phi$ possibly held"), Def $\stackrel{d b}{\rightarrow} \Phi$ (" $\Phi$ definitely held"), and Inst $\Phi$ (" $\Phi$ definitely held in a specific global state"). This paper defines these modalities and gives efficient algorithms for detecting them. The algorithms are based on algorithms of Garg and Waldecker, Alagar and Venkatesan, Cooper and Marzullo, and Fromentin and Raynal. Complexity analysis shows that under reasonable assumptions, these real-time-clock-based detection algorithms are less expensive than detection algorithms based on Lamport's happened-before ordering. Sample applications are given to illustrate the benefits of this approach.
\end{abstract}

Key words: global predicate detection, consistent global states, distributed debugging, realtime monitoring

\section{Introduction}

A history of a distributed system can be modeled as a sequence of events. Since execution of a particular sequence of events leaves the system in a well-defined global state, a history uniquely determines the sequence of global states through which the system has passed. Unfortunately, in a distributed system without perfect clock synchronization, it is, in general, impossible for a process to determine the order in which events on different processors actually occurred. Therefore, no process can determine the sequence of global states through which the system passed. This leads to an obvious difficulty for detecting whether a global state predicate (hereafter simply called a "predicate") held.

\footnotetext{
*A preliminary description of this work appeared in [32]. The author gratefully acknowledges the support of NSF under CAREER Award CCR-9876058 and the support of ONR under Grant N00014-99-1-0358. Email: stoller@cs.indiana.edu Web: http://www.cs.indiana.edu/ ${ }^{\text {stoller / }}$
} 
Cooper and Marzullo proposed a solution for asynchronous distributed systems [6]. Their solution involves two modalities, which we denote by Poss $\stackrel{h b}{\rightarrow}$ ("possibly") and Def $\stackrel{h b}{\rightarrow}$ ("definitely"). These modalities are based on logical time [19] as embodied in the happened-before relation $\stackrel{h b}{\rightarrow}$, a partial ordering ${ }^{1}$ of events that reflects potential causal dependencies. Happened-before is not a total order, so it does not uniquely determine the history, but it does restrict the possibilities. Given a predicate $\Phi$, a computation satisfies Poss $\stackrel{h b}{\rightarrow} \Phi$ iff there is some interleaving of events that is consistent with happened-before and in which the system passes through a global state satisfying $\Phi$. A computation satisfies Def $\stackrel{h b}{\rightarrow} \Phi$ iff for every interleaving of events that is consistent with happened-before, the system passes through a global state satisfying $\Phi$.

Cooper and Marzullo's definitions of these modalities established an important conceptual framework for predicate detection in asynchronous systems, which has been the basis for considerable research $[8,13,4,18,34,14,5,12]$. In practice, though, Poss $\stackrel{h b}{\rightarrow}$, Def $^{\stackrel{h b}{\rightarrow}}$, and other modalities based on happened-before have significant drawbacks in many cases. First, in many systems, it is difficult to determine the happened-before relation. Happened-before can be determined if each process maintains a vector clock. This requires that a vector timestamp with $O(N)$ components be attached to every message, where $N$ is the number of processes in the system, and imposes computational overhead of $O(N)$ operations per message received (to update the vector clock). Generating code that inserts and removes the vector timestamps without changing the existing types in the programs (which would open a can of worms) or copying entire messages (which is inefficient) can be difficult. If the programs use a stream-oriented communication protocol that does not provide message boundaries, such as TCP, the difficulty is significantly compounded, since a receiver might receive a fragment of a "message" or several "messages" in a single receive event. Furthermore, piggybacking vector timestamps requires changing all communication statements in the application, even if the predicate of interest involves the state of only one module. If source code is not available for part of the system, this might be impossible. Happened-before can be determined without vector clocks, if all processes inform the monitor of all send events and receive events and provide the monitor with enough information to determine the correspondence between send and receive events (i.e., for each receive event, the monitor can determine which send event sent the received message). However, this method often has significant drawbacks, too. In general, determining the correspondence between send and receive events requires piggybacking an identifier (e.g., a sequence number) on each message; this involves the same difficulties as piggybacking a vector timestamp. ${ }^{2}$ An additional drawback of this method is that the monitor must be informed of all send events and receive events. With vector timestamps (or if the happened-before relation is not needed), it suffices to inform the monitor only of events that might change the truth value of the predicate of interest $;^{3}$ this can significantly reduce the amount of information sent to the

\footnotetext{
${ }^{1}$ In this paper, all partial orderings are irreflexive unless specified otherwise.

${ }^{2}$ Even if the underlying communication protocol uses sequence numbers, operating-system protection mechanisms may prevent the monitoring system from accessing them.

${ }^{3}$ This optimization is not explicitly incorporated in our algorithms, but that is easily done.
} 
monitor.

A second drawback of detecting Poss $\stackrel{h b}{\rightarrow} \Phi$ or $\operatorname{Def}^{\stackrel{h b}{\rightarrow}} \Phi$ is the computational cost: the worst-case time complexity is $\Omega\left(E^{N}\right)$, where $E$ is the maximum number of events executed by any process. The worst case occurs when there is little or no communication and hence few causal dependencies, so that many interleavings must be explored. For example, this exponential cost was seen in two sample applications considered in [33], namely, a coherence protocol and a spanning-tree algorithm. A third drawback is that, in systems with hidden channels [2] (i.e., means of communication other than messages), happened-before does not accurately capture causal relationships, so Poss $\stackrel{h b}{\rightarrow} \Phi$ and Def $^{\stackrel{h b}{\rightarrow}} \Phi$ do not accurately capture the meanings of "possibly held" and "definitely held".

This paper proposes a framework for predicate detection in systems with approximately-synchronized real-time clocks. Timestamps from these clocks can be used to define two orderings on events: $\stackrel{d b}{\rightarrow}$ ("definitely occurred before") and $\stackrel{p b}{\rightarrow}$ ("possibly occurred before"). By (roughly speaking) substituting each of these orderings for happened-before in the definitions of Poss $\stackrel{h b}{\rightarrow}$ and Def $\stackrel{h b}{\rightarrow}$, we obtain definitions of four new modalities. The two modalities based on $\stackrel{d b}{\rightarrow}$ are closely analogous to Poss $\stackrel{h b}{\rightarrow}$ and Def $\stackrel{h b}{\rightarrow}$, so we denote them by Poss $\stackrel{d b}{\rightarrow}$ and Def $\stackrel{d b}{\rightarrow}$. We obtain algorithms for detecting Poss $\stackrel{d b}{\rightarrow}$ and Def $\stackrel{d b}{\rightarrow}$ by adapting algorithms of Garg and Waldecker [13, 14], Alagar and Venkatesan [1], and Cooper and Marzullo [6]. Modalities based on $\stackrel{p b}{\rightarrow}$ are quite different, because $\stackrel{p b}{\rightarrow}$ (unlike $\stackrel{h b}{\rightarrow}$ and $\stackrel{d b}{\rightarrow}$ ) is not a partial ordering. In fact, $\stackrel{p b}{\rightarrow}$ yields a degenerate case, in which the analogues of Poss $^{\stackrel{h b}{\rightarrow}}$ and Def $\stackrel{h b}{\rightarrow}$ are equivalent. We show that this single modality, which we denote by Inst, is closely related to Fromentin and Raynal's concept of Properly $\stackrel{h b}{\rightarrow}[9,10]$, and we adapt for detecting Inst an algorithm of theirs for detecting Properly $\stackrel{h b}{\rightarrow}$.

Our detection framework is applicable to a wide range of systems, since it does not require that clocks be synchronized to within a fixed bound. However, the quality of clock synchronization does affect the two event orderings just described and therefore the results of detection. For example, consider Inst $\Phi$. Informally, a computation satisfies Inst $\Phi$ iff the timestamps imply that there was an instant during the computation when predicate $\Phi$ held, i.e., iff there is some collection of local states that form a global state satisfying $\Phi$ and that, based on the timestamps, definitely overlapped in time. Suppose $\Phi$ actually holds in a global state $g$ that persists for time $\delta$. Whether Inst $\Phi$ holds depends on the quality of synchronization. Roughly, if the maximum difference between clocks is known to be less than $\delta$, then Inst $\Phi$ holds; otherwise, there is in some cases no way to determine whether the local states in $g$ actually overlapped in time, so Inst $\Phi$ might not hold.

The quality of clock synchronization affects also the cost of detection. For example, consider Poss $\stackrel{d b}{\rightarrow} \Phi$. Informally, a computation satisfies Poss $\stackrel{d b}{\rightarrow} \Phi$ iff there is some collection of local states that form a global state satisfying $\Phi$ and that, based on the timestamps, possibly overlapped in time. The larger the error in clock synchronization, the more combinations of local states possibly overlap. In general, $\Phi$ must be evaluated in each such combination of local states. Thus, the larger this error, the more expensive the detection. If this error is bounded relative to the mean interval between relevant events (i.e., events that potentially truthify or falsify $\Phi$ ), then the number of global states that must be checked is linear in $E$. In the asynchronous case, the number of global 
states that must be checked is $O\left(E^{N}\right)$.

The above condition on the error in clock synchronization holds in many systems. In most localarea distributed systems, protocols like NTP can efficiently maintain synchronization of clocks to within a few milliseconds [28]. Even in extremely wide-area distributed systems like the Internet, clock synchronization can usually be maintained to within a few tens of milliseconds [26, 28]. The detection framework and algorithms proposed here are designed to provide a basis for monitoring and debugging applications in such systems. Some sample applications are described in Section 7, including applications in which clocks and timers provide a hidden channel, causing detection based on happened-before to be less appropriate. These examples also illustrate that the clocks are available to the monitored processes as well as the detection algorithm.

Directions for future work include: implementing the detection algorithms described above; developing efficient algorithms for detecting global properties that depend explicitly on time; and investigating clock-based detection of sequences of global states, perhaps along the lines of temporal modalities based on happened-before [17, 3, 11].

\section{Related Work}

Marzullo and Neiger [24] discuss global property detection in partially-synchronous systems in which a fixed bound $\epsilon$ on the error between clocks is known. In the notation of this paper, they define modalities Poss $\stackrel{h d}{\rightarrow}$ and Def $\stackrel{h d}{\rightarrow}$, where $\stackrel{h d}{\longrightarrow} \triangleq \stackrel{d b}{\rightarrow} \cup \stackrel{h b}{\rightarrow}$, and give detection algorithms for these two modalities. Combining happened-before and real-time ordering exploits more information about the computation and hence can reduce the number of explored global states (compared to using either ordering alone). This is certainly desirable whenever it is feasible. Modifying the algorithms in this paper to take happened-before into account is a straightforward exercise, and the resulting algorithms would be appropriate for monitoring some systems. This paper presents algorithms that do not use happened-before for three reasons. First and most important, as discussed in Section 1, it is often difficult in practice to modify a system so that the monitor can determine the happenedbefore relation; consequently, detection algorithms that depend on happened-before have limited applicability. ${ }^{4}$ Second, not using happened-before enables some optimizations (specifically, those involving priority queues) that are impossible if causal ordering is also used. Third, incorporating happened-before would have obscured the presentation and complexity analysis of the real-timebased parts of the algorithms, which are the novel parts.

Contributions of this paper relative to [24] include: detection algorithms based purely on realtime clocks; more efficient detection algorithms; and definition of an algorithm for Inst. [24] does not consider any modality analogous to Inst. Also, [24] assumes a fixed bound on the error in clock synchronization. Our framework allows that bound to vary over time; this supports tighter

\footnotetext{
${ }^{4}$ Our algorithms apply directly even to programs that use high-level communication libraries (e.g., a distributed shared memory (DSM) library) for which source code is not available; detecting happened-before in such cases would be difficult.
} 
bounds hence more accurate monitoring results.

An attractive feature of Properly $\stackrel{h b}{\rightarrow}[9,10]$ and Inst is that the monitor can report a single global state $g$ satisfying $\Phi$ that the system actually passed through. Def does not have this feature. However, Properly $\stackrel{h b}{\rightarrow}$ gives useful information only about systems that perform global (i.e., system-wide) barrier synchronization. Such synchronization is expensive and rarely used. In contrast, assuming reasonably good clock synchronization, Inst is informative even in the absence of barrier synchronization. Since Inst, like Properly $\stackrel{h b}{\rightarrow}$, can be detected efficiently for arbitrary predicates, it appears to be a useful modality.

The "possibly occurred before" relation $\stackrel{p b}{\longrightarrow}$ is reminiscent of Lamport's "can affect" relation for concurrent systems [21, 22]. Both relations may contain cycles because of overlap: for $\stackrel{p b}{\rightarrow}$, overlap of interval timestamps; for "can affect", overlap of non-atomic events. Our framework assumes events are atomic; this is appropriate for systems with message-based communication.

Veríssimo [35] discusses the uncertainty in event orderings caused by the granularity ${ }^{5}$ and imperfect synchronization of digital real-time clocks, analyzes the conditions under which this uncertainty is significant for an application, and describes a synchronization technique, suitable for certain applications, that masks this uncertainty. However, [35] does not aim for a general approach to detecting global properties in the presence of this uncertainty.

Mayo and Kearns [25] present two detection algorithms for distributed systems with partiallysynchronized clocks. Both algorithms assume a fixed upper bound on the difference between clocks; they neither tolerate worse clock synchronization under bad conditions nor exploit better clock synchronization under good conditions. The first algorithm attempts to determine whether a predicate holds at a specified instant; our algorithms are designed for continuous monitoring. The second algorithm detects Inst $\Phi$ with worst-case time complexity $O\left(N^{2} E\right)$, compared to $O((N \log N) E)$ for our algorithm in Section 6.

\section{Background}

A computation of a single process is called a local computation and is represented as a finite or infinite sequence of local states and events. Thus, a local computation has the form

$$
e_{1}, s_{1}, e_{2}, s_{2}, e_{3}, s_{3}, \ldots
$$

where the $e_{\alpha}$ are events, and the $s_{\alpha}$ are local states. By convention, $e_{1}$ corresponds to creation of the process. If the sequence is finite, it ends with an event that corresponds (by convention) to the termination of the process.

A computation of a distributed system is a collection of local computations, one per process. A computation is represented as a function from process names to local computations. We use

\footnotetext{
${ }^{5}$ Our framework accommodates the granularity of digital clocks by using $\leq$ instead of $<$ in TS1 and TS2, introduced in Section 3.
} 
integers $1,2, \ldots, N$ as process names; thus, for a computation $c$, the local computation of process $i$ is $c(i)$. Variables $i$ and $j$ always range over process names. We use $E v(c)$ and $S t(c)$ to denote the sets of all events and all local states, respectively, in a computation $c$. For convenience, we assume that all events and local states in a computation are distinct.

The following functions are implicitly parameterized by a computation; the computation being considered should be evident from the context. For an event $e, \operatorname{pr}(e)$ denotes the process on which $e$ occurs. For a local state $s, \operatorname{pr}(s)$ denotes the process that passes through $s$, and $\mathcal{S}(s)$ and $\mathcal{T}(s)$ denote the start event and terminal event, respectively, of $s$. For example, for a computation containing local computation (1), $\mathcal{S}\left(s_{2}\right)$ is $e_{2}$, and $\mathcal{T}\left(s_{2}\right)$ is $e_{3}$.

A global state of a distributed system is a collection of local states, one per process, represented as a function from process names to local states. The set of global states of a computation $c$ is denoted $G S(c)$; thus, $g$ is in $G S(c)$ iff for each process $i, g(i)$ is a local state in $c(i)$. We define a reflexive partial ordering $\preceq$ on global states by:

$$
g \preceq g^{\prime} \triangleq\left(\forall i: g(i)=g^{\prime}(i) \vee g(i) \text { occurs before } g^{\prime}(i)\right)
$$

All of the orderings defined in this paper, including $\preceq$, are implicitly parameterized by a computation; the computation being considered should be evident from the context.

Each event $e$ has an interval timestamp its(e), which is an interval with lower endpoint lwr $(e)$ and upper endpoint upr(e). Our results apply to any totally-ordered time domain, regardless of whether it is continuous or discrete. Thus, the interval could be a range of integers or a range of real numbers. We model events as being atomic and instantaneous; the width of the interval timestamp depends only on the quality of clock synchronization when the event occurs. We assume that the interval timestamps are non-decreasing and are consistent with the order of events; more precisely, we assume:

TS1. For every event $e, \operatorname{lwr}(e) \leq u p r(e)$.

TS2. For every event $e_{1}$ with an immediately succeeding event $e_{2}$ on the same process, $\operatorname{lwr}\left(e_{1}\right) \leq$ $\operatorname{lwr}\left(e_{2}\right)$ and $\operatorname{upr}\left(e_{1}\right) \leq \operatorname{upr}\left(e_{2}\right)$.

TS3. For every event $e_{1}$ and every event $e_{2}$, if $e_{1}$ occurred before $e_{2}$ in real-time, then $\operatorname{lwr}\left(e_{1}\right) \leq$ $\operatorname{upr}\left(e_{2}\right)$.

There are various ways of satisfying these assumptions, depending on the underlying clock synchronization mechanism. As a simple (yet realistic) example, if the clock synchronization algorithm never decreases the value of a clock, and if all of the machines (more precisely, all of the machines relevant to the predicate being detected) synchronize to a single time server, then TS1-TS3 hold if timestamps are chosen such that the value of the time server's clock was in the interval its $(e)$ when event $e$ occurred. Thus, a machine can take $\operatorname{lwr}(e)=t-\varepsilon$ and $u p r(e)=t+\varepsilon$, where $t$ is the value of its local clock when $e$ occurred, and $\varepsilon$ is a bound on the difference between its clock and the time server's clock when $e$ occurred. In systems in which time servers are organized in peer 
groups or in a client-server hierarchy, its $(e)$ can be determined from an appropriate combination of the bounds on the errors between the relevant clocks. In either case, the information needed to construct interval timestamps can be obtained from standard clock synchronization subsystems, such as NTP $[27,28]$ or the Distributed Time Service in OSF DCE [29].

\section{Generic Theory of Consistent Global States}

Predicate detection in asynchronous systems is based on the theory of consistent global states (CGSs) [2]. Informally, a global state is consistent if it could have occurred during the computation. It is convenient to define "consistent" in terms of ideals. Recall that an ideal of a partial order $\langle S, \prec\rangle$ is a set $I \subseteq S$ such that $(\forall x \in I: \forall y \in S: y \prec x \Rightarrow y \in I)$. Ideals of $\langle E v(c), \stackrel{h b}{\rightarrow}\rangle$ are called consistent cuts [2]. Recall that for any partial order, the set of its ideals ordered by inclusion $(\subseteq)$ forms a lattice [8]. Furthermore, the set of CGSs ordered by $\preceq$ forms a lattice that is isomorphic to the lattice of consistent cuts [30,2]. This isomorphism has an important consequence for detection algorithms: it implies that a minimal increase with respect to $\preceq$ corresponds to advancing one process by one event (because adjacent ideals of $\langle E v(c), \stackrel{h b}{\rightarrow}\rangle$ differ by exactly one event) and hence that the lattice of CGSs can be explored by repeatedly advancing one process by one event. This principle underlies detection algorithms of Cooper and Marzullo [6], Garg and Waldecker [13, 14], and Alagar and Venkatesan [1].

In this section, we show that the above theory is not specific to the happened-before relation but rather applies to any partial ordering $\hookrightarrow$ on events, provided $\hookrightarrow$ is process-wise-total, i.e., for any two events $e_{1}$ and $e_{2}$ on the same process, if $e_{1}$ occurred before $e_{2}$, then $e_{1} \hookrightarrow e_{2}$. This generalized theory underlies the detection algorithms in Sections 5 and 6.

Definition of CGSs. Let $c$ be a computation, and let $\hookrightarrow$ be a relation on $E v(c)$. We define a relation $\rightsquigarrow \hookrightarrow$ on $S t(c)$, with the informal interpretation: $s \rightsquigarrow \hookrightarrow s^{\prime}$ if $s$ ends before $s^{\prime}$ starts. Formally,

$$
s \rightsquigarrow s^{\prime} \triangleq \begin{cases}\mathcal{S}(s) \hookrightarrow \mathcal{S}\left(s^{\prime}\right) & \text { if } \operatorname{pr}(s)=\operatorname{pr}\left(s^{\prime}\right) \\ \mathcal{T}(s) \hookrightarrow \mathcal{S}\left(s^{\prime}\right) & \text { if } \operatorname{pr}(s) \neq \operatorname{pr}\left(s^{\prime}\right) .\end{cases}
$$

Two local states are concurrent with respect to $\hookrightarrow$ if they are not related by $\rightsquigarrow \hookrightarrow$. A global state is consistent with respect to $\hookrightarrow$ if its constituent local states are pairwise concurrent:

$$
\operatorname{consis}^{\hookrightarrow}(g) \triangleq(\forall i, j: i \neq j \Rightarrow \neg(g(i) \rightsquigarrow \hookrightarrow g(j)))
$$

Thus, the set of CGSs of computation $c$ with respect to $\hookrightarrow$ is

$$
C G S^{\hookrightarrow}(c)=\left\{g \in G S(c) \mid \operatorname{consis}^{\hookrightarrow}(g)\right\}
$$

Note that $C G S^{\frac{h b}{\rightarrow}}$ is the usual notion of CGSs. 
Definitions of Poss and Def. The modalities Poss $\stackrel{h b}{\rightarrow}$ and Def $\stackrel{h b}{\rightarrow}$ for asynchronous systems are defined in terms of the lattice $\langle C G S \stackrel{h b}{\rightarrow}(c), \preceq\rangle$. We generalize them as follows.

A computation $c$ satisfies Poss $\hookrightarrow \Phi$ iff $C G S^{\hookrightarrow}(c)$ contains a global state satisfying $\Phi$.

Def $\hookrightarrow$ is defined in terms of paths. A path through a partial order $\langle S, \preceq\rangle$ is a finite or infinite sequence $^{6} \sigma$ of distinct elements of $S$ such that: (i) $\sigma[1]$ is minimal with respect to $\preceq$; (ii) for all $\alpha \in \operatorname{domain}(\sigma)$, if $\alpha<|\sigma|$, then $\sigma[\alpha+1]$ is an immediate successor ${ }^{7}$ of $\sigma[\alpha]$; and (iii) if $\sigma$ is finite, then $\sigma[|\sigma|]$ is maximal with respect to $\preceq$. Informally, each path through $\left\langle C G S^{\hookrightarrow}(c), \preceq\right\rangle$ corresponds to an order in which the events in the computation could have occurred.

A computation $c$ satisfies $\operatorname{Def}^{\hookrightarrow} \Phi$ iff every path through $\left\langle C G S^{\hookrightarrow}(c), \preceq\right\rangle$ contains a global state satisfying $\Phi$.

CGSs and Ideals. When $\hookrightarrow$ is a process-wise-total partial ordering, there is a natural correspondence between $C G S^{\hookrightarrow}(c)$ and ideals of $\langle E v(c), \hookrightarrow\rangle$. One can think of an ideal $I$ as the set of events that have occurred. Executing a set $I$ of events leaves each process $i$ in the local state immediately following the last event of process $i$ in $I$. Thus, ideal $I$ corresponds to the global state $g$ such that for all $i, \mathcal{S}(g(i))$ is the maximal element of $\{e \in I \mid \operatorname{pr}(e)=i\}$. This correspondence is an isomorphism.

Theorem 1. For every process-wise-total partial ordering $\hookrightarrow$ on $E v(c)$, the partial order $\langle C G S \hookrightarrow(c), \preceq$ \rangle is a lattice and is isomorphic to the lattice of ideals of $\langle E v(c), \hookrightarrow\rangle$.

Proof. This is true for the same reasons as in the standard theory based on happened-before $[30,2,8]$. The proof is straightforward.

The following corollary underlies the detection algorithms in Sections 5 and 6 .

Corollary 2. For any process-wise-total partial ordering $\hookrightarrow$, if global state $g^{\prime}$ is an immediate successor of $g$ in $\left\langle C G S^{\hookrightarrow}(c), \preceq\right\rangle$, then the ideal corresponding to $g^{\prime}$ contains exactly one more event than the ideal corresponding to $g$.

Proof. This follows from Theorem 1 and the fact that for any partial order $S$, if one ideal of $S$ is an immediate successor of another ideal of $S$, then those two ideals differ by exactly one element.

\subsection{Instantiations of $\hookrightarrow$}

This paper focuses on two instantiations of the generic theory of CGSs. In Section $5, \hookrightarrow$ is instantiated with the ordering $\stackrel{d b}{\rightarrow}$ ("definitely occurred before"), defined by

$$
e_{1} \stackrel{d b}{\rightarrow} e_{2} \triangleq \begin{cases}e_{1} \text { occurs before } e_{2} & \text { if } \operatorname{pr}\left(e_{1}\right)=\operatorname{pr}\left(e_{2}\right) \\ \operatorname{upr}\left(e_{1}\right)<\operatorname{lwr}\left(e_{2}\right) & \text { if } \operatorname{pr}\left(e_{1}\right) \neq \operatorname{pr}\left(e_{2}\right)\end{cases}
$$

\footnotetext{
${ }^{6}$ We use 1-based indexing for sequences.

${ }^{7}$ For a reflexive or irreflexive partial order $\langle S, \prec\rangle$ and elements $x \in S$ and $y \in S, y$ is an immediate successor of $x$ iff $x \neq y \wedge x \prec y \wedge \neg(\exists z \in S \backslash\{x, y\}: x \prec z \wedge z \prec y)$.
} 
This ordering cannot be defined solely in terms of the timestamps its $\left(e_{1}\right)$ and $i t s\left(e_{2}\right)$, because TS1 and TS2 allow consecutive events on a process to have identical timestamps. Therefore, we assume that a process records a local sequence number as well as an interval timestamp for each event. The following theorem, together with Corollary 2, implies that detection algorithms for Poss $\stackrel{d b}{\rightarrow}$ and Def $\stackrel{d b}{\rightarrow}$ can explore all elements of $C G S \stackrel{d b}{\rightarrow}$ by repeatedly advancing one process by one event.

Theorem 3. For every computation $c, \stackrel{d b}{\rightarrow}$ is a process-wise-total partial ordering on $E v(c)$.

Proof. It follows immediately from the definitions that $\stackrel{d b}{\rightarrow}$ is process-wise-total. We need to show that $\stackrel{d b}{\longrightarrow}$ is irreflexive, acyclic, and transitive. Irreflexivity is obvious. For transitivity, we suppose $e_{1} \stackrel{d b}{\rightarrow} e_{2}$ and $e_{2} \stackrel{d b}{\rightarrow} e_{3}$, and show $e_{1} \stackrel{d b}{\rightarrow} e_{3}$. First consider the case $\operatorname{pr}\left(e_{1}\right)=\operatorname{pr}\left(e_{3}\right)$. In this case, it suffices to show that $e_{1}$ occurred before $e_{3}$. If $\operatorname{pr}\left(e_{2}\right)=\operatorname{pr}\left(e_{1}\right)$, then the desired result follows from transitivity of "occurred before". If $\operatorname{pr}\left(e_{2}\right) \neq \operatorname{pr}\left(e_{1}\right)$, then using TS1, the hypothesis $e_{1} \stackrel{d b}{\rightarrow} e_{2}$, TS1 again, and finally the hypothesis $e_{2} \stackrel{d b}{\rightarrow} e_{3}$, we have the chain of inequalities $\operatorname{lwr}\left(e_{1}\right) \leq \operatorname{upr}\left(e_{1}\right)<\operatorname{lwr}\left(e_{2}\right) \leq \operatorname{upr}\left(e_{2}\right)<\operatorname{lwr}\left(e_{3}\right)$, so $\operatorname{lwr}\left(e_{1}\right)<\operatorname{lwr}\left(e_{3}\right)$, so by TS2, $e_{1}$ occurred before $e_{3}$. Next consider the case $\operatorname{pr}\left(e_{1}\right) \neq \operatorname{pr}\left(e_{3}\right)$. Note that $\neg\left(\operatorname{pr}\left(e_{1}\right)=\operatorname{pr}\left(e_{2}\right) \wedge p r\left(e_{2}\right)=\operatorname{pr}\left(e_{3}\right)\right)$. If $\operatorname{pr}\left(e_{2}\right) \neq \operatorname{pr}\left(e_{1}\right)$, then it is easy to show (by case analysis on whether $\operatorname{pr}\left(e_{2}\right)=\operatorname{pr}\left(e_{3}\right)$ ) that $\operatorname{upr}\left(e_{1}\right)<\operatorname{lwr}\left(e_{2}\right) \leq \operatorname{lwr}\left(e_{3}\right)$, so $\operatorname{upr}\left(e_{1}\right)<\operatorname{lwr}\left(e_{3}\right)$, as desired. If $\operatorname{pr}\left(e_{2}\right) \neq \operatorname{pr}\left(e_{3}\right)$, then it is easy to show (by case analysis on whether $\left.\operatorname{pr}\left(e_{2}\right)=\operatorname{pr}\left(e_{1}\right)\right)$ that $\operatorname{upr}\left(e_{1}\right) \leq \operatorname{upr}\left(e_{2}\right)<\operatorname{lwr}\left(e_{3}\right)$, so $u p r\left(e_{1}\right)<\operatorname{lwr}\left(e_{3}\right)$, as desired.

Given transitivity, to conclude acyclicity, it suffices to show that there are no cycles of size 2. We suppose $e_{1} \stackrel{d b}{\rightarrow} e_{2}$ and $e_{2} \stackrel{d b}{\rightarrow} e_{1}$, and derive a contradiction. If $\operatorname{pr}\left(e_{1}\right)=\operatorname{pr}\left(e_{2}\right)$, then the fact that "occurred before" is a total order on the events of each process yields the desired contradiction. If $\operatorname{pr}\left(e_{1}\right) \neq \operatorname{pr}\left(e_{2}\right)$, then using TS1, the hypothesis $e_{1} \stackrel{d b}{\rightarrow} e_{2}$, TS1 again, and finally the hypothesis $e_{2} \stackrel{d b}{\rightarrow} e_{1}$, we obtain the chain of inequalities $\operatorname{lwr}\left(e_{1}\right) \leq \operatorname{upr}\left(e_{1}\right)<\operatorname{lwr}\left(e_{2}\right) \leq \operatorname{upr}\left(e_{2}\right)<\operatorname{lwr}\left(e_{1}\right)$, which implies $\operatorname{lwr}\left(e_{1}\right)<\operatorname{lwr}\left(e_{1}\right)$, a contradiction.

In Section $6, \hookrightarrow$ is instantiated with the ordering $\stackrel{p b}{\rightarrow}$ ("possibly occurred before"), defined by

$$
e_{1} \stackrel{p b}{\rightarrow} e_{2} \triangleq \neg\left(e_{2} \stackrel{d b}{\rightarrow} e_{1}\right)
$$

As discussed in Section $6, \stackrel{p b}{\rightarrow}$ is not a partial ordering, so we detect modalities based on $\stackrel{p b}{\rightarrow}$ by relating them to a modality based on $\stackrel{d b}{\rightarrow}$.

Another interesting possibility, mentioned in Section 2 but not further pursued in this paper, is to instantiate $\hookrightarrow$ with $\stackrel{d b}{\longrightarrow} \cup \stackrel{h b}{\longrightarrow}$.

\section{Detection Based on a Strong Event Ordering: Poss $\stackrel{d b}{\rightarrow}$ and Def $\stackrel{d b}{\rightarrow}$}

By the discussion in Section $4, \stackrel{d b}{\rightarrow}$ induces a notion $C G S \stackrel{d b}{\rightarrow}$ of CGSs. If $g \in C G S \stackrel{d b}{\rightarrow}(c)$, then the local states in $g$ possibly overlapped in time. For example, Figure 1 shows a computation $c_{1}$ and the lattice $\left.\left\langle C G S \stackrel{d b}{\rightarrow}\left(c_{1}\right)\right), \preceq\right\rangle$. The pair of arcs enclosing each event show the endpoints of the interval 
timestamp. In the lattice, a node labeled $i, j$ represents the global state in which process 1 is local state $s_{i}^{1}$ and process 2 is in local state $s_{j}^{2}$.
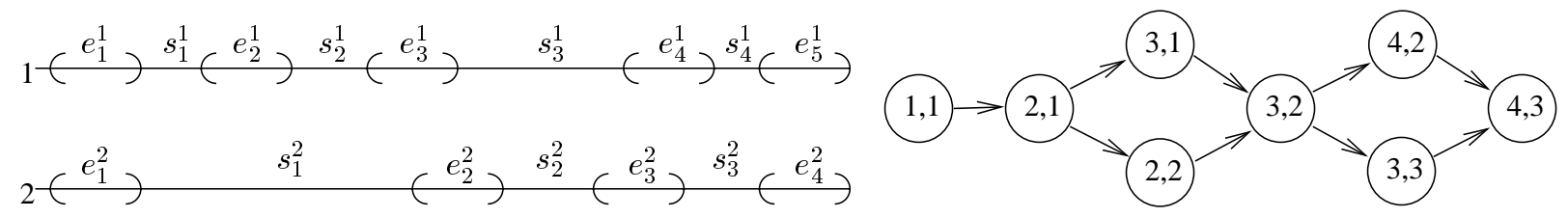

Figure 1: Left: A computation $c_{1}$. Right: The lattice $\left\langle C G S \stackrel{d b}{\rightarrow}\left(c_{1}\right), \preceq\right\rangle$.

We consider in this paper only detection algorithms with a passive monitor. Each process in the original system sends its timestamped local states to a new process, called the monitor. More specifically, when a process executes an event, thereby terminating its current local state $s$, the process sends to the monitor a message containing $s$ and the timestamps its $(\mathcal{S}(s))$ and $i t s(\mathcal{T}(s)) .{ }^{8}$

We consider only on-line detection, in which the monitor detects the property as soon as possible. Algorithms for off-line detection, in which the monitor waits until the computation has terminated before checking whether a property holds, can be obtained as special cases. We consider first algorithms for detecting Poss $\stackrel{d b}{\rightarrow}$ for a restricted class of predicates and then consider general algorithms for detecting Poss $\stackrel{d b}{\rightarrow}$ and $\operatorname{Def}^{\stackrel{d b}{\rightarrow}}$.

\subsection{Algorithms for Poss $\stackrel{d b}{\stackrel{b}{b}}$ and Def $\stackrel{d b}{\stackrel{d b}{b}}$ for Conjunctive Predicates}

A predicate is conjunctive if it is a conjunction of predicates, each of which depends on the local state of at most one process. For example, if $x_{i}$ is a local variable of process $i$, then the predicate $x_{1}>0 \wedge x_{2}<0$ is conjunctive, and the predicate $x_{1}>x_{2}$ is not conjunctive. Garg and Waldecker [13] and Hurfin et al. [16] developed efficient algorithms for detecting Poss $\stackrel{h b}{\rightarrow} \Phi$ for conjunctive predicates $\Phi$. Garg and Waldecker also developed an efficient algorithm for detecting Def $\stackrel{h b}{\rightarrow}$ for such predicates [14]. Garg and Waldecker's algorithms can be adapted in a straightforward way to detect Poss $\stackrel{d b}{\rightarrow}$ and Def $\stackrel{d b}{\rightarrow}$ for such predicates, by (roughly) replacing comparisons based on happened-before with comparisons based on $\stackrel{d b}{\rightarrow}$. This yields detection algorithms with worst-case time complexity $O\left(N^{2} E\right)$, where $E$ is the maximum number of events executed by any process. The worst-case time complexity of both algorithms can be reduced to $O((N \log N) E)$ by exploiting the total ordering on numbers.

We start by reviewing Garg and Waldecker's algorithm for detecting Poss $\stackrel{h b}{\rightarrow} \Phi$ for conjunctive predicates. Suppose the predicate of interest is $\Phi=\bigwedge_{i=1}^{N} \phi_{i}$, where $\phi_{i}$ depends on the local state of

\footnotetext{
${ }^{8}$ Several straightforward optimizations are possible. For example, each message might describe only the differences between consecutive reported local states, rather than repeating the entire local state. Also, except for the initial local state, it suffices to include with local state $s$ only the timestamp its $(\mathcal{T}(s)$ ), since its $(\mathcal{S}(s))$ was sent in the previous message to the monitor. Also, for a given predicate $\Phi$, events that cannot possibly truthify or falsify $\Phi$ can be ignored.
} 
process $i$. Each process $i$ sends to the monitor timestamped local states satisfying $\phi_{i}$; local states not satisfying $\phi_{i}$ are not reported. For each process $i$, the monitor maintains a queue $q_{i}$ and adds each timestamped local state received from process $i$ to the end of $q_{i}$. Let head $(q)$ denote the head of a non-empty queue $q$. If for some $i$ and $j$, head $\left(q_{i}\right) \stackrel{h b}{\rightarrow} \operatorname{head}\left(q_{j}\right)$, then head $\left(q_{i}\right)$ is removed from $q_{i}$. The heads of the queues are repeatedly compared and sometimes removed in this way, until the heads of the non-empty queues are pairwise concurrent. At that point, if all the queues are nonempty, then the heads of the queues form a CGS satisfying $\Phi$, so the algorithm returns that CGS (thereby indicating that Poss $\stackrel{h b}{\rightarrow} \Phi$ holds); if some queue is empty, then the monitor waits to receive more local states and then repeats the procedure just described. The worst-case time complexity is $O\left(N^{2} E\right)$, because there are $O(N E)$ local states, and each time a local state is removed from $q_{i}$, the new head of $q_{i}$ is compared with the heads of the other $O(N)$ queues.

Our algorithm for detecting Poss $\stackrel{d b}{\rightarrow} \bigwedge_{i=1}^{N} \phi_{i}$ appears in Figure 2. If the computation satisfies Poss $\stackrel{d b}{\rightarrow} \Phi$, then the algorithm returns a CGS satisfying $\Phi$. The first line in the body of the while loop is justified as follows. Expanding the definition (4) of consis $\stackrel{d b}{\rightarrow}(g)$ yields

$$
(\forall i, j: i \neq j \Rightarrow \operatorname{upr}(\mathcal{T}(g(i))) \geq \operatorname{lwr}(\mathcal{S}(g(j)))) .
$$

Using the fact that for all $i, \operatorname{upr}(\mathcal{T}(\operatorname{head}(g(i)))) \geq \operatorname{lwr}(\mathcal{S}($ head $(g(i))))$, which follows from TS1 and TS2, one can show that (8) is equivalent to

$$
\min _{i}(\operatorname{upr}(\mathcal{T}(\operatorname{head}(g(i))))) \geq \max _{i}(\operatorname{lwr}(\mathcal{S}(\operatorname{head}(g(i)))))
$$

From this, one can show that, if the global state $\left(\lambda i \operatorname{head}\left(q_{i}\right)\right)$ is not consistent, then in order to find a CGS, some process $i$ such that $\operatorname{upr}\left(\mathcal{T}\left(\right.\right.$ head $\left.\left.\left(q_{i}\right)\right)\right)$ is minimal must be advanced.

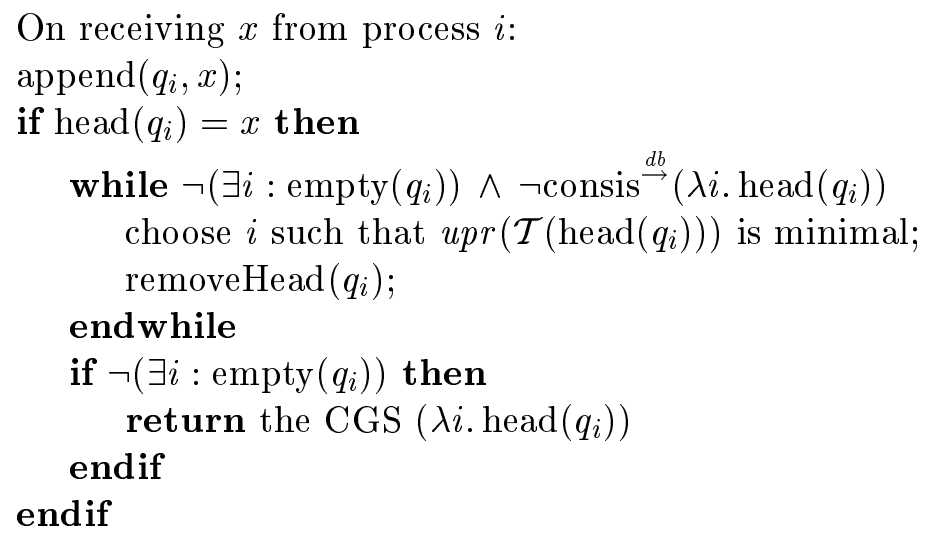

Figure 2: Algorithm PossConjAlg $(\Phi)$ for detecting Poss $\stackrel{d b}{\rightarrow} \Phi$ for conjunctive predicates. Process $i$ sends to the monitor only local states satisfying its local predicate.

We now describe some optimizations to the basic algorithm. To evaluate (9) efficiently, we 
modify the algorithm to maintain two priority queues $p_{1}$ and $p_{2}$, whose contents are determined by the invariants:

I1: For each process $i$ such that $q_{i}$ is non-empty, $p_{1}$ contains a record with key $\operatorname{lwr}\left(\mathcal{S}\left(\right.\right.$ head $\left.\left.\left(q_{i}\right)\right)\right)$ and satellite data $i . p_{1}$ contains no other records.

I2: For each process $i$ such that $q_{i}$ is non-empty, $p_{2}$ contains a record with key $\operatorname{upr}\left(\mathcal{T}\left(\right.\right.$ head $\left.\left.\left(q_{i}\right)\right)\right)$ and satellite data $\langle i, p t r\rangle$, where $p t r$ is a pointer to the record with satellite data $i$ in $p_{1} . p_{2}$ contains no other records.

Whenever the head of a queue changes, $p_{1}$ and $p_{2}$ are updated in $O(\log N)$ time to maintain these invariants. Recall that the operations on a priority queue $p$ include getMin $(p)$, which returns a record $\langle k, d\rangle$ with key $k$ and satellite data $d$ such that $k$ is minimal, and $\operatorname{extractMin}(p)$, which removes and returns such a record. We also use priority queues with analogous operations based on maximal key values. Using I1 and I2, (9) is equivalent to

$$
\operatorname{key}\left(\operatorname{get} \operatorname{Min}\left(p_{2}\right)\right) \geq \operatorname{key}\left(\operatorname{get} \operatorname{Max}\left(p_{1}\right)\right)
$$

where $\operatorname{key}(\langle k, d\rangle)=k$. Thus, in Figure 2 , consis $\stackrel{d b}{\rightarrow}\left(\lambda i\right.$. head $\left.\left(q_{i}\right)\right)$ can be replaced with (10), and line $(\dagger)$ can be replaced with $\langle k,\langle i, p t r\rangle\rangle:=\operatorname{extractMin}\left(p_{2}\right)$.

To evaluate $\left(\exists i: \operatorname{empty}\left(q_{i}\right)\right)$ efficiently, we modify the algorithm to maintain a natural number numEmpty, whose value is determined by the invariant: numEmpty $=\left|\left\{i \mid \operatorname{empty}\left(q_{i}\right)\right\}\right|$. Whenever a queue is modified, numEmpty is updated in constant time to maintain this invariant. In Figure 2, both occurrences of $\left(\exists i\right.$ : empty $\left.\left(q_{i}\right)\right)$ can be replaced with numEmpty $>0$.

Let PossConjAlg denote the optimized algorithm. To analyze its time complexity, recall that an operation on a priority queue containing $n$ records takes $O(\log n)$ time. A constant number of such operations are performed for each local state, so the worst-case time complexity of PossConjAlg is $O(E N \log N)$. Note that the time complexity is independent of the quality of clock synchronization.

The algorithm in [14] for detecting $\operatorname{Def}^{\stackrel{h b}{\rightarrow}} \Phi$ for conjunctive $\Phi$ can be adapted in a similar way to detect $\operatorname{Def}^{\stackrel{d b}{\rightarrow}} \Phi$ for such predicates.

\subsection{General Algorithm for Poss $\stackrel{d b}{\rightarrow}$}

We develop an on-line detection algorithm for Poss $\stackrel{d b}{\rightarrow} \Phi$ by adapting Alagar and Venkatesan's algorithm for detecting Poss $\stackrel{h b}{\rightarrow} \Phi$ in non-terminating (i.e., infinite) computations [1]. Their algorithm is based on their procedure for depth-first search of a lattice of CGSs. A depth-first exploration of the lattice of CGSs for an infinite computation would never backtrack and thus would never visit some CGSs near the beginning of the lattice. So, in their algorithm, the lattice is divided into a sequence of sublattices $L_{0}, L_{1}, L_{2}, \ldots$, corresponding to increasing prefixes of the computation, and depth-first search is used to explore each sublattice $L_{i+1}-L_{i}$. The following paragraphs describe how to adapt their algorithm to on-line detection of Poss $\stackrel{d b}{\rightarrow} \Phi$. 
Finding the initial CGS. In the asynchronous setting, the initial CGS simply contains the initial local state of each process. In the timed setting, that global state might not be consistent, since the processes might have been started at different times.

Theorem 4. For every computation $c$, if $C G S^{\hookrightarrow}(c)$ is not empty, then $\left\langle C G S^{\hookrightarrow}(c), \preceq\right\rangle$ contains a unique minimal element, i.e., $c$ has a unique initial CGS.

Proof. The existence of minimal elements in the lattice of CGSs follows immediately from nonemptiness and the absence of infinite descending chains in $\preceq[7$, chapter 2]. We prove by contradiction that the lattice of CGSs has a unique minimal element. Suppose CGSs $g_{1}$ and $g_{2}$ are minimal and $g_{1} \neq g_{2}$. Let $\wedge_{G}$ denote the meet operation of the lattice of CGSs. Note that $g_{1} \npreceq g_{2}$ (by the assumptions that $g_{2}$ is minimal and $\left.g_{1} \neq g_{2}\right)$ and $\left(g_{1} \wedge_{G} g_{2}\right) \preceq g_{2}$ (by the definition of $\left.\wedge_{G}\right)$, so $\left(g_{1} \wedge_{G} g_{2}\right) \neq g_{1}$. By definition of $\wedge_{G},\left(g_{1} \wedge_{G} g_{2}\right) \preceq g_{1}$, which together with $\left(g_{1} \wedge_{G} g_{2}\right) \neq g_{1}$ contradicts the assumed minimality of $g_{1}$.

To find the initial CGS, we exploit the fact that for every conjunctive predicate $\Phi$, if a computation satisfies Poss $\stackrel{d b}{\rightarrow} \Phi$, then PossConjAlg $(\Phi)$ finds and returns the unique minimal CGS satisfying $\Phi$; the proof of this is closely analogous to the proof of the corresponding property of Garg and Waldecker's algorithm [14]. A corollary is: if $C G S \stackrel{d b}{\rightarrow}(c)$ is not empty, then PossConjAlg(true) returns the initial CGS (otherwise, PossConjAlg(true) never calls return). ${ }^{9}$

Choosing the sequence of sublattices. To avoid delays in detection, when the monitor receives a timestamped local state, it constructs the largest CGS $g_{2}$ that can be constructed from the local states it has received so far; this is done by the while loop in Figure 4. This CGS implicitly defines the next sublattice $L_{i+1}: L_{i+1}$ contains exactly the CGSs $g$ such that $g \preceq g_{2}$. Let $g_{1}$ denote the CGS constructed when the previous local state was received, i.e., the CGS corresponding to sublattice $L_{i}$. After constructing $g_{2}$, the monitor does a depth-first search of the sublattice $L_{i+1}-L_{i}$, which (by definition) contains CGSs $g$ such that $g_{1} \preceq g \preceq g_{2}$.

Exploration of a sublattice. There are two main steps in the exploration of the sublattice of CGSs between a CGS $g_{1}$ and a larger CGS $g_{2}$ :

- Use a procedure initStates $\left(g_{1}, g_{2}\right)$ to compute the set $S$ of minimal (with respect to $\preceq$ ) CGSs in that sublattice.

- For each CGS $g$ in $S$, use a procedure depthFirstSearch $\left(g, g_{1}, g_{2}\right)$ to do a depth-first search starting from $g$ of a fragment of that sublattice. These searches together explore the entire sublattice.

Alagar and Venkatesan observed that initStates can be computed efficiently as follows. For a local state $s$, let minstate $(s)$ be the unique minimal CGS containing $s$, and let $\operatorname{succ}(s)$ be the local state

\footnotetext{
${ }^{9}$ Strictly speaking, the argument of PossConjAlg should have the form $\bigwedge_{i=1}^{N} \phi_{i}$. We use PossConjAlg $(\operatorname{true})$ as an abbreviation for PossConjAlg $\left(\bigwedge_{i=1}^{N}\right.$ true $)$.
} 
that occurs immediately after $s$ on the same process (if there is no such local state, then $\operatorname{succ}(s)$ is undefined). Then initStates $\left(g_{1}, g_{2}\right)$ is given by [1]:

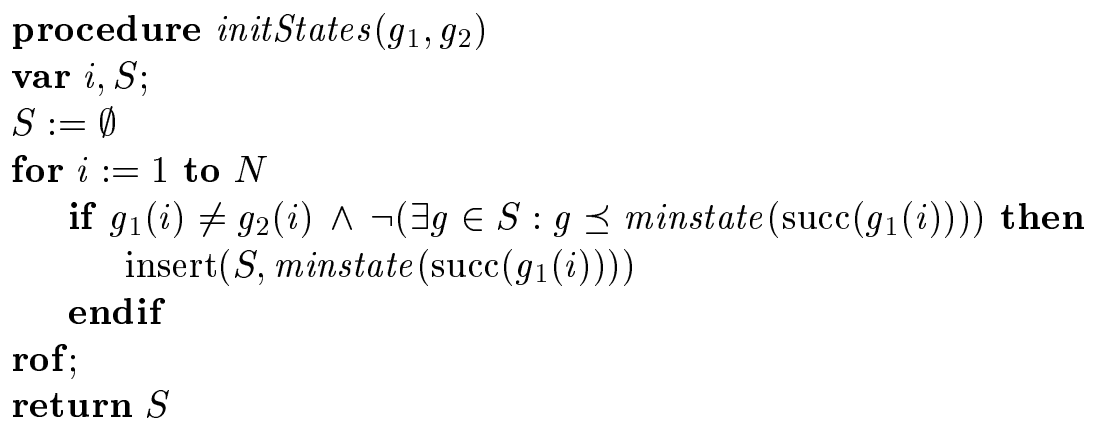

Computing minstate. Our algorithm for computing minstate is similar to our algorithm for computing the initial CGS. It relies on the following property of PossConjAlg: if PossConjAlg $(\Phi)$ is started from a global state $g$ (i.e., for all $i$, local states of process $i$ that occur before $g(i)$ are ignored), and if the remainder of the computation satisfies Poss $\stackrel{d b}{\rightarrow} \Phi$, then PossConjAlg $(\Phi)$ finds the unique minimal CGS greater than $g$ and satisfying $\Phi$. For a global state $g$ and a local state $s$, let $g[i \mapsto s]$ denote the global state that is the same as $g$ except that the local state of process $i$ is $s$. A simple way to compute minstate $(s)$ is to call PossConjAlg(true) starting from the global state $g_{0}[\operatorname{pr}(s) \mapsto s]$, where $g_{0}$ is the initial CGS. An optimization is sometimes possible. Consider a call minstate $\left(s_{2}\right)$. If minstate has not previously been called with a local state of $\operatorname{pr}\left(s_{2}\right)$ as argument, then the optimization does not apply, and minstate is computed as described above. Otherwise, let $s_{1}$ be the argument in the previous call to minstate on a local state of $\operatorname{pr}\left(s_{2}\right)$. Observe that $s_{1}$ occurred before $s_{2}$, because: (1) minstate is called only from initStates, and initStates is called on a non-decreasing chain of CGSs (this is a property of the algorithm in Figure 4 below), and (2) (assuming short-circuiting evaluation of $\wedge$ in initStates) initStates $\left(g_{1}, g_{2}\right)$ calls minstate $\left(\operatorname{succ}\left(g_{1}(i)\right)\right)$ only if $g_{1}(i) \neq g_{2}(i)$. Since $s_{1}$ occurred before $s_{2}$, minstate $\left(s_{1}\right) \preceq$ minstate $\left(s_{2}\right)$. So, we can start PossConjAlg(true) from global state minstate $\left(s_{1}\right)[\operatorname{pr}(s) \mapsto s]$ instead of $g_{0}[\operatorname{pr}(s) \mapsto s]$. This leads to the following algorithm. For each $i$, old $(i)$ contains the result of the previous call to minstate on a local state of process $i$; initially, old $(i)$ is set to $g_{0}$.

procedure minstate $(s)$

$\operatorname{old}(\operatorname{pr}(s)):=$ the CGS returned by PossConjAlg $(\operatorname{true})$ started from old $(\operatorname{pr}(s))[\operatorname{pr}(s) \mapsto s]$; return old $(p r(s))$

Depth-first search of fragment of sublattice. Since a CGS may have multiple predecessors in the lattice of CGSs, the search algorithm needs (for efficiency) some mechanism to ensure that each CGS is explored only once. A straightforward approach is to maintain a set containing the CGSs that have been visited so far. However, this may be expensive in both space and time. Alagar and Venkatesan [1] proposed the following clever alternative. Introduce a total ordering $<_{i d x}$ on CGSs, defined by: $g_{1}<_{i d x} g_{2}$ if $\operatorname{index}\left(g_{1}\right)$ is lexicographically smaller than index $\left(g_{2}\right)$, where for a 
global state $g, \operatorname{index}(g)$ is the tuple $\left\langle k_{1}, \ldots, k_{n}\right\rangle$ such that $g(i)$ is the $k_{i}$ 'th local state of process $i$. During the depth-first search, explore a CGS $g$ only from the immediate predecessor (with respect to $\preceq$ ) of $g$ that is maximal (among the immediate predecessors of $g$ ) with respect to $<_{i d x}$. This leads to the algorithm in Figure 3, where for a CGS $g, \operatorname{pred}(g)$ is the set of immediate predecessors of $g$ in the lattice of CGSs. To compute $\operatorname{pred}(g)$ : for each process $i$, check whether moving process $i$ back by one local state yields a CGS, and if so, include the resulting CGS in the set.

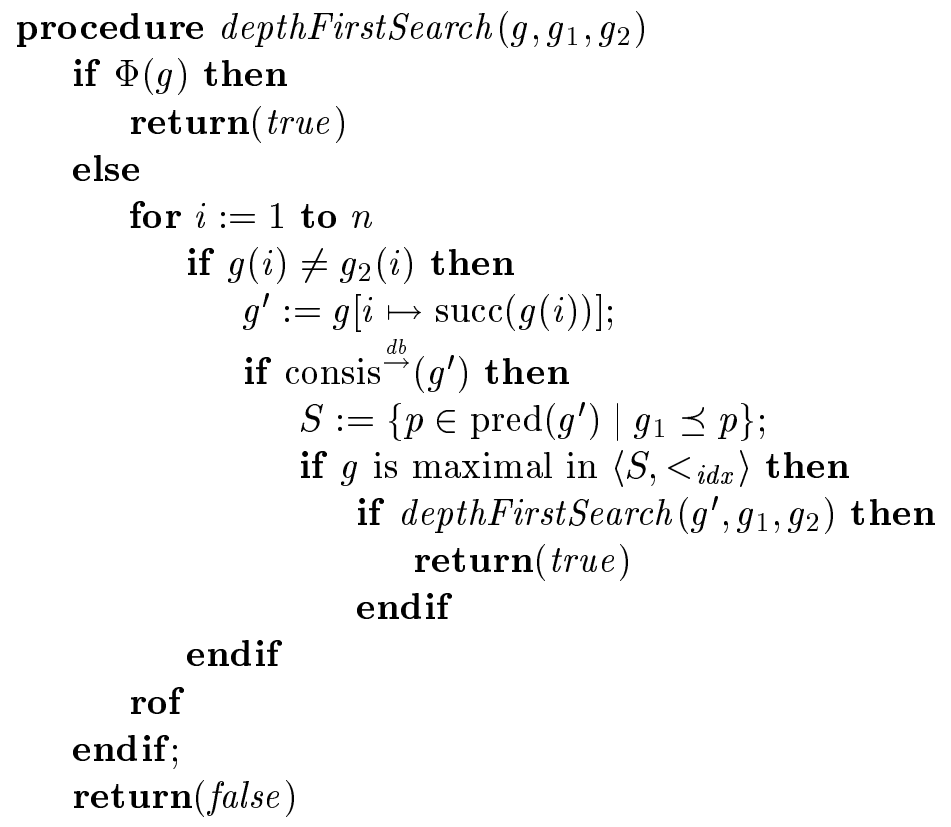

Figure 3: Algorithm for depth-first search.

Putting these pieces together yields the on-line detection algorithm in Figure 4. All local states "after" $g_{0}$ (i.e., local states $s$ such that $g_{0}(\operatorname{pr}(s)$ ) occurs before $s$ ) are handled by the "On receiving $s$ from process $i$ " statement, even though some of these local states might have been received before the initial CGS was found.

Recall that a process sends a local state to the monitor when that local state ends. This is natural (because $\rightsquigarrow \stackrel{d b}{\rightarrow}$ depends on when local states end) but can delay detection. One approach to bounding and reducing this delay is for a process that has not reported an event to the monitor recently to send a message to the monitor to report that it is still in the same local state (as if reporting execution of skip). Another approach, described in [24], requires a bound on message latency: at each instant, the monitor can use its own local clock and this bound to determine a lower bound on the ending time of the last local state it received from a process. 
Initialization Phase :

$g_{0}:=$ the CGS returned by PossConjAlg $($ true $) ; \quad\left(* g_{0}\right.$ is the initial CGS $\left.*\right)$

$g_{2}:=g_{0}$;

For all local states $x$ such that $g_{0}(\operatorname{pr}(x))$ occurs before $x$ :

On receiving $x$ from process $i$ :

append $\left(q_{i}, x\right)$;

$g_{1}:=g_{2}$;

while $\left(\exists j: \neg \operatorname{empty}\left(q_{j}\right) \wedge\right.$ consis $\left.\stackrel{d b}{\rightarrow}\left(g_{2}\left[j \mapsto \operatorname{head}\left(q_{j}\right)\right]\right)\right) \quad(*$ construct largest CGS $*)$

$g_{2}(j):=\operatorname{head}\left(q_{j}\right)$

$\operatorname{removeHead}\left(q_{j}\right)$

endwhile;

for $s$ in initStates $\left(g_{1}, g_{2}\right)$

if depthFirstSearch $\left(g, g_{1}, g_{2}\right)$ then

report Poss $\stackrel{d b}{\rightarrow} \Phi$ and exit

endif

rof

Figure 4: Algorithm for detecting Poss $\stackrel{d b}{\stackrel{\rightarrow}{\rightarrow}} \Phi$.

\subsubsection{Complexity}

To analyze the time complexity, we consider separately the cost of all invocations of minstate and the cost of all other operations. In effect, for each process, the calls to minstate cause PossConjAlg algorithm to be executed $N$ times (once for each process) on (at worst) the entire computation. Thus, the total cost of calls to PossConjAlg from minstate, and hence the total cost of calls to minstate, is $O\left(E N^{2} \log N\right)$. The total cost of all executions of the while loop in Figure 4 is $O\left(E N^{3}\right)$, since: (1) evaluating the loop condition takes time $O\left(N^{2}\right)$, and the condition is evaluated at most once for each of the $O(N E)$ local states; (2) the loop body is executed at most once for each of the $O(N E)$ local states, and each execution takes constant time. The total cost of all executions of the body of the for loop in Figure 4 is $O\left(\left|C G S^{\stackrel{d b}{\rightarrow}}(c)\right| N^{2}\right)$, since the depth-first search takes $O\left(N^{2}\right)$ time per CGS, since evaluating pred takes $O\left(N^{2}\right)$ time. Each call to initStates takes $O\left(N^{3}\right)$ time (excluding the cost of calls to minstate), because: (1) evaluating $\preceq$ takes $O(N)$ time, and (2) $\preceq$ may be evaluated $O\left(N^{2}\right)$ times, because of the for loop and the existential quantifier. The total cost of all calls to initStates is $O\left(N^{4} E\right)$, since initStates is called at most once per local state. Summing these contributions, we conclude that the worst-case time complexity of the algorithm is $O\left(\left|C G S^{\stackrel{d b}{\rightarrow}}(c)\right| N^{2}+E N^{4}\right)$.

$\left|C G S^{\stackrel{d b}{\rightarrow}}(c)\right|$ depends on the rate at which events occur relative to the error between clocks. To simplify the complexity analysis, suppose: (1) the interval between consecutive events at a process is at least $\tau,(2)$ the error between clocks is known to be at most $\varepsilon$, and (3) the interval timestamp on an event $e$ is given by $\operatorname{lwr}(e)=t-\varepsilon$ and $\operatorname{upr}(e)=t+\varepsilon$, where $t$ is the value of 
the local clock of machine $\operatorname{pr}(e)$ when $e$ occurred. Then, for every event $e, \operatorname{upr}(e)-\operatorname{lwr}(e)=2 \varepsilon$. If $\tau>2 \varepsilon$, then each local state is concurrent with at most 3 local states of each other process, so each local state is in at most $O\left(3^{N-1}\right)$ CGSs, so there are $O\left(3^{N} E\right)$ CGSs, so the worst-case time complexity of the algorithm is $O\left(3^{N} E N^{2}\right)$. If $\tau \leq 2 \varepsilon$, then each local state is concurrent with at most $\lceil(4 \varepsilon+\tau) / \tau\rceil+1$ local states of each other process, so there are $O\left((\lceil 4 \varepsilon / \tau\rceil+2)^{N-1} E\right)$ CGSs, so the worst-case time complexity of the algorithm is $O\left((\lceil 4 \varepsilon / \tau\rceil+2)^{N-1} E N^{2}\right)$. In both cases, the worst-case time complexity of detecting Poss $\stackrel{d b}{\rightarrow}$ is linear in $E$, which is normally much larger than $N$; in contrast, the worst-case time complexity of general algorithms for detecting Poss $\stackrel{h b}{\rightarrow}$ and $\operatorname{Def}^{\stackrel{h b}{\rightarrow}}$ is $\Omega\left(E^{N}\right)$.

A more realistic complexity analysis requires considering distributions of inter-event times, rather than simply fixing a minimum value. Specifically, we consider distributed computations with inter-event times selected from a normal (i.e., Gaussian) distribution with mean $\mu$ and standard deviation $\sqrt{\mu}$ (negative numbers selected from the distribution were ignored). For simplicity, we continue to assume a fixed bound $\varepsilon$ on the error between clocks. The number of CGSs then depends on $N, E$, and the ratio $\mu / \varepsilon$. As in the cases analyzed above, the number of CGSs scales linearly with $E$; this is illustrated by the graph in Figure 5. Figure 6 plots the number of CGSs vs. $\mu / \varepsilon$ and $N$. One can see that when $\mu / \varepsilon$ is large, the number of CGSs increases slowly (roughly linearly) with $N$; when $\mu / \varepsilon$ is small, the number of CGSs increases exponentially with $N$.

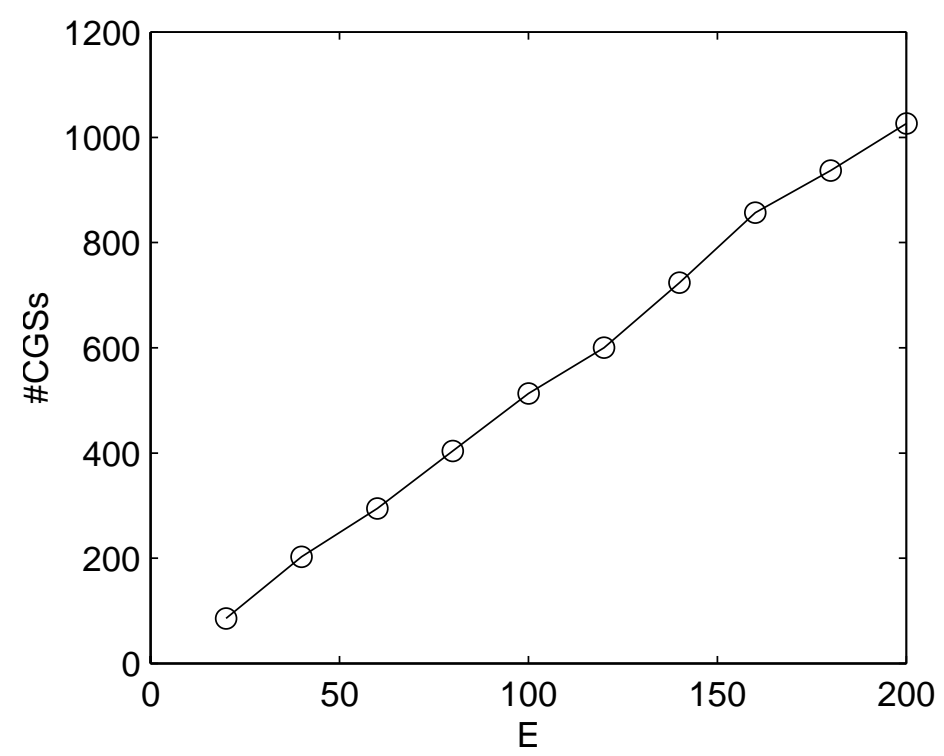

Figure 5: Number of CGSs vs. $E$, for $\mu / \varepsilon=10$ and $N=4$. 

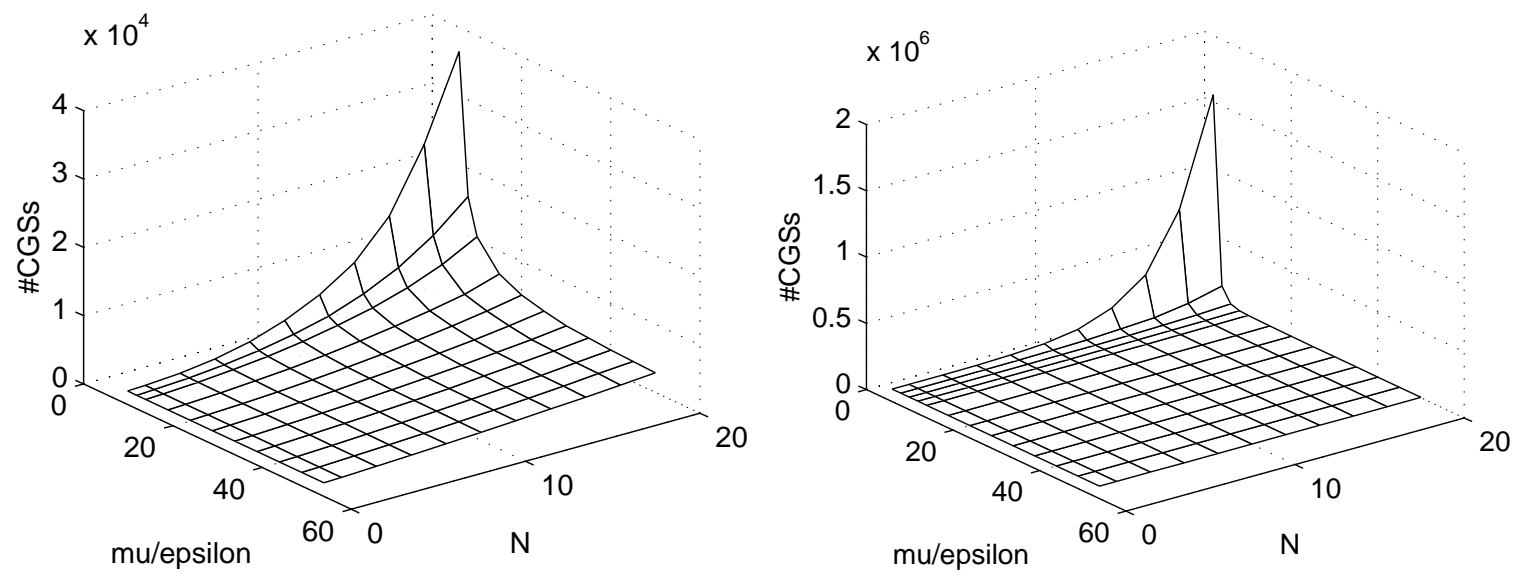

Figure 6: Left: Number of CGSs vs. $\mu / \varepsilon$ and $N$, for $E=100$, with $\mu / \varepsilon$ ranging from 6 to 50 . Right: Number of CGSs vs. $\mu / \varepsilon$ and $N$, for $E=100$, with $\mu / \varepsilon$ from 2 to 50. Note that the vertical scales in these two graphs are very different.

\subsection{General Algorithm for Def $\stackrel{d b}{\rightarrow}$}

The detection algorithm for $\operatorname{Def}^{\stackrel{h b}{\rightarrow}} \Phi$ in $[6,24]$ can be adapted to detect $\operatorname{Def}^{\stackrel{d b}{\rightarrow}} \Phi$ by (roughly) replacing each condition of the form $e_{1} \stackrel{h b}{\rightarrow} e_{2}$ with $e_{1} \stackrel{d b}{\rightarrow} e_{2}$. That algorithm divides the lattice into levels. The level of a local state in a local computation is the number of local states preceding it in that computation. The level of a global state $g$ is the sum of the levels of the constituent local states. Level $\ell$ of the lattice of CGSs contains the CGSs with level $\ell$. Following [6, 24], we give an algorithm in which the monitor constructs one level of the lattice of CGSs at a time. Constructing one level of the lattice at a time is unnecessary and sometimes delays detection of a property; this construction is used only to simplify the presentation.

The algorithm used by the monitor to detect $\operatorname{Def}^{\stackrel{d b}{\rightarrow}} \Phi$ is given in Figure 7 . The lowest level of the lattice contains only the initial CGS. The while loop maintains the following invariant: last contains CGSs that are reachable from the initial CGS without passing through a CGS satisfying $\Phi$. In line $(\dagger)$ of the algorithm, the monitor considers each global state $g$ in last and each process $i$, and checks whether the local state $\operatorname{succ}(g(i))$ is concurrent with the local states in $g$ of all the other processes. (The monitor waits for the local states $\operatorname{succ}(g(1)), \ldots, \operatorname{succ}(g(N))$, if they have not already arrived.) If so, the monitor adds $g[i \mapsto \operatorname{succ}(g(i))]$ to current if it is not already in current.

Since $\Phi$ could be false in all CGSs, and because we assume that the cost of evaluating $\Phi$ on each global state is a constant, the worst-case asymptotic time complexity of this algorithm equals the worst-case asymptotic time complexity of constructing $C G S \stackrel{d b}{\rightarrow}$. For each CGS, the algorithm advances each of the $N$ processes and, if the resulting global state $g$ is consistent, the algorithm checks whether $g$ is already in current. Let $T_{m}$ denote the total cost of these membership checks; then constructing $C G S \stackrel{d b}{\rightarrow}(c)$ takes $\Theta\left(|C G S \stackrel{d b}{\rightarrow}(c)| N^{2}+T_{m}\right)$ time. $T_{m}$ depends on the data structure 


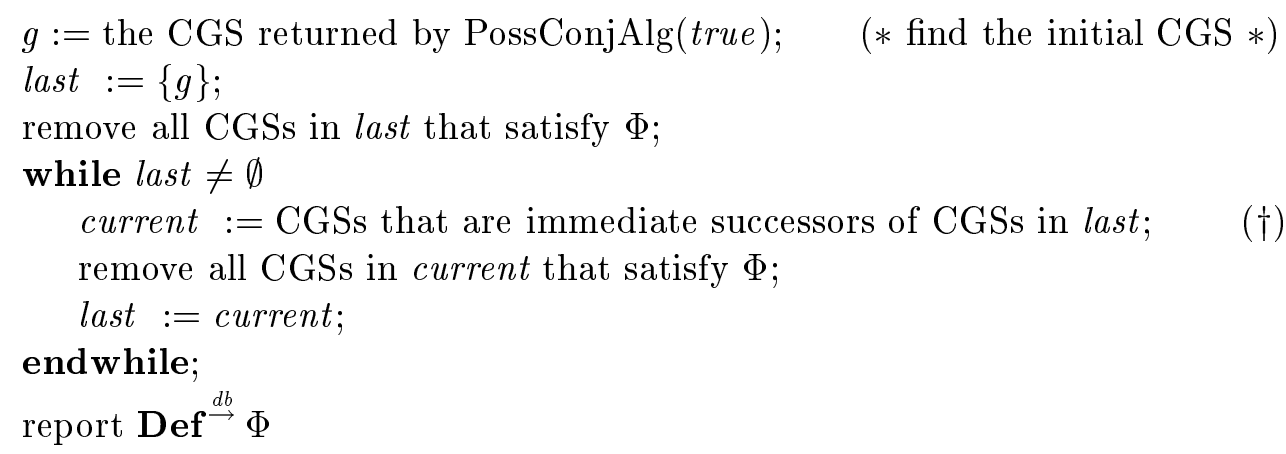

used to implement current. With a naive array-based implementation, each check has constant cost, so $T_{m}$ is $\Theta\left(E^{N}\right)$, due to the cost of initializing the arrays, so the worst-case time complexity of the algorithm is $\Theta\left(E^{N} N^{2}\right) .{ }^{10}$

However, this implementation has a serious disadvantage: the time complexity remains $\Omega\left(E^{N}\right)$ even if the actual number of CGSs is much smaller than $E^{N}$, which is typically the case. Thus, generally preferable alternatives are to implement current as a dictionary, using a hash table or balanced trees. Let $W(c)$ be the width of the lattice $C G S \stackrel{d b}{\rightarrow}(c)$, i.e., the maximum size of a level of that lattice. If balanced trees are used, each membership check has cost $O(\log W(c))$, so the worst-case time complexity is $O\left(\left|C G S^{\stackrel{d b}{\rightarrow}}(c)\right|\left(N^{2}+N \log W(c)\right)\right)$.

Both $|C G S \stackrel{d b}{\rightarrow}(c)|$ and $W(c)$ depend on the rate at which events occur relative to the error between clocks. To simplify the complexity analysis, we introduce $\tau$ and $\varepsilon$, with the same meanings as in Section 5.2.1. If $\tau>2 \varepsilon$, then there are $O\left(3^{N} E\right)$ CGSs (as above), and $W(c)$ is $O\left(3^{N}\right)$, so the worst-case time complexity of the algorithm is $O\left(3^{N} E N^{2}\right)$. If $\tau \leq 2 \varepsilon$, then there are $O\left((\lceil 4 \varepsilon / \tau\rceil+2)^{N-1} E\right)$ CGSs (as above), and $W(c)$ is $O\left((\lceil 4 \varepsilon / \tau\rceil+2)^{N-1}\right)$, so the worst-case time complexity is $O\left((\lceil 4 \varepsilon / \tau\rceil+2)^{N-1} E N^{2} \log (\lceil 4 \varepsilon / \tau\rceil+2)\right)$. In both cases, the worst-case time complexity of detecting Def $\stackrel{d b}{\rightarrow}$ is linear in $E$; in contrast, the worst-case time complexity of general algorithms for detecting $\operatorname{Def}^{h b}$ is $\Omega\left(E^{N}\right)$.

For a more realistic complexity analysis, we consider the same distribution of inter-event times and the same bound on error between clocks as in the last paragraph of Section 5.2. Of course, the number of CGSs is still characterized by Figures 5 and 6 . It is easy to argue that under these assumptions, the expected size of each level is independent of $E$ and depends in the same fashion as the number of CGSs on $\mu / \varepsilon$ and $N$. Thus, graphs showing the dependence of $W(c)$ on $\mu / \varepsilon$ and $N$ would have the same shape as the graphs in Figure 6.

\footnotetext{
${ }^{10}$ In on-line detection, $E$ is not known in advance, so the arrays may need to be resized (e.g., doubled) occasionally. This does not change the asymptotic time complexity.
} 


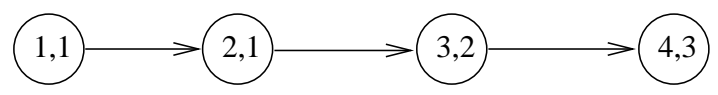

Figure 8: The lattice $\left\langle C G S^{\stackrel{p b}{\rightarrow}}\left(c_{1}\right), \preceq\right\rangle$.

\section{Detection Based on a Weak Event Ordering: Inst}

Using (3), $\stackrel{p b}{\rightarrow}$, defined in (7), induces a relation $\rightsquigarrow \stackrel{p b}{\rightarrow}$ on local states, with the interpretation: $s \rightsquigarrow \stackrel{p b}{\rightarrow} s^{\prime}$ if $s$ possibly ended before $s^{\prime}$ started. Two local states are strongly concurrent if they are not related

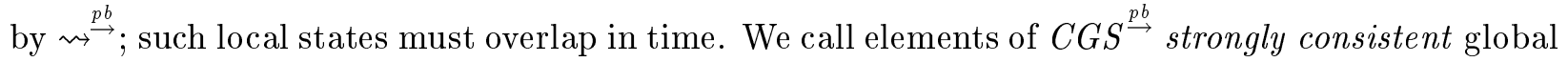
states (SCGSs). ${ }^{11}$ For example, Figure 8 shows $\left\langle C G S^{\stackrel{p b}{\rightarrow}}\left(c_{1}\right), \preceq\right\rangle$; recall that computation $c_{1}$ is shown in Figure 1. Note that $\left\langle C G S \stackrel{p b}{\rightarrow}\left(c_{1}\right), \preceq\right\rangle$ is a total order. More generally, we can show:

Theorem 5. For all computations $c,\left\langle C G S^{\stackrel{p b}{\rightarrow}}(c), \preceq\right\rangle$ is a total order and therefore a lattice.

Proof. Suppose not, i.e., suppose there exist a computation $c$, two global states $g, g^{\prime} \in C G S \stackrel{p b}{\rightarrow}(c)$, and two processes $i$ and $j$ such that $g(i) \rightsquigarrow \stackrel{p b}{\rightarrow} g^{\prime}(i)$ and $g^{\prime}(j) \leadsto \stackrel{p b}{\rightarrow} g(j)$. By definition of $C G S^{\stackrel{p b}{\rightarrow}}(c)$, $\neg(g(i) \rightsquigarrow \stackrel{p b}{\rightarrow} g(j))$, so $\operatorname{upr}(\mathcal{S}(g(j)))<\operatorname{lwr}(\mathcal{T}(g(i)))$. By hypothesis, $g(i) \stackrel{p b}{\mapsto} g^{\prime}(i)$, so $\operatorname{lwr}(\mathcal{T}(g(i))) \leq$ $\operatorname{upr}\left(\mathcal{S}\left(g^{\prime}(i)\right)\right)$, so by transitivity, upr $(\mathcal{S}(g(j)))<\operatorname{upr}\left(\mathcal{S}\left(g^{\prime}(i)\right)\right)$. By definition of $C G S \stackrel{p b}{\rightarrow}(c), \neg\left(g^{\prime}(j) \rightsquigarrow \stackrel{p b}{\rightarrow}\right.$ $\left.g^{\prime}(i)\right)$, so $\operatorname{upr}\left(\mathcal{S}\left(g^{\prime}(i)\right)\right)<\operatorname{lwr}\left(\mathcal{T}\left(g^{\prime}(j)\right)\right)$, so by transitivity, $\operatorname{upr}(\mathcal{S}(g(j)))<\operatorname{lwr}\left(\mathcal{T}\left(g^{\prime}(j)\right)\right)$. By hypothesis, $g^{\prime}(j) \rightsquigarrow \stackrel{p b}{\rightarrow} g(j)$, so $\operatorname{lwr}\left(\mathcal{T}\left(g^{\prime}(j)\right)\right) \leq \operatorname{upr}(\mathcal{S}(g(j)))$, so by transitivity, upr $(\mathcal{S}(g(j)))<$ $\operatorname{upr}(\mathcal{S}(g(j)))$, which is a contradiction.

It follows that Poss $\stackrel{p b}{\rightarrow}$ and Def $\stackrel{p b}{\rightarrow}$ are equivalent, i.e., for all computations $c$ and predicates $\Phi, c$ satisfies Poss $\stackrel{p b}{\rightarrow} \Phi$ iff $c$ satisfies Def $\stackrel{p b}{\rightarrow} \Phi$. We define Inst ("instantaneously") to denote this modality (i.e., Poss $\stackrel{p b}{\rightarrow}$ and Def $\stackrel{p b}{\rightarrow}$ ). Informally, a computation satisfies Inst $\Phi$ if there is a global state $g$ satisfying $\Phi$ and such that the system definitely passes through $g$ during the computation.

Theorem 1 does not apply to $\stackrel{p b}{\longrightarrow}$, because:

Lemma 6. $\stackrel{p b}{\rightarrow}$ is not a partial ordering.

Proof. Consider the computation in Figure 9. The actual orderings between $e_{2}^{2}$ and $e_{2}^{1}$ and between $e_{2}^{2}$ and $e_{3}^{1}$ cannot be determined from the interval timestamps, so $e_{3}^{1} \stackrel{p b}{\rightarrow} e_{2}^{2}$ and $e_{2}^{2} \stackrel{p b}{\rightarrow} e_{2}^{1}$. Since also $e_{2}^{1} \stackrel{p b}{\rightarrow} e_{3}^{1}, \stackrel{p b}{\rightarrow}$ contains a cycle.

In light of this, it is not surprising that a minimal increase in $\left\langle C G S^{\stackrel{p b}{\rightarrow}}(c), \preceq\right\rangle$ does not necessarily correspond to advancing one process by one event. For example, consider the computation $c_{1}$ in Figure 1. As shown in Figure 8, two processes advance between the second and third SCGSs of $c_{1}$. In some computations, a minimal increase $\langle C G S \stackrel{p b}{\rightarrow}(c), \preceq\rangle$ corresponds to an advance of multiple

\footnotetext{
${ }^{11}$ Fromentin and Raynal call elements of $C G S^{\stackrel{h b}{\longrightarrow}}$ inevitable global states [10].
} 
events per process. Such a computation $c_{2}$ is shown in Figure 9. There is no local state of process 2 with which $s_{2}^{1}$ definitely overlaps, so $s_{2}^{1}$ is not part of any SCGS, and process 1 advances by two events between consecutive SCGSs of $c_{2}$. Computation $c_{2}$ has only two SCGSs: $\left\langle s_{1}^{1}, s_{1}^{2}\right\rangle$ and $\left\langle s_{3}^{1}, s_{2}^{2}\right\rangle$.

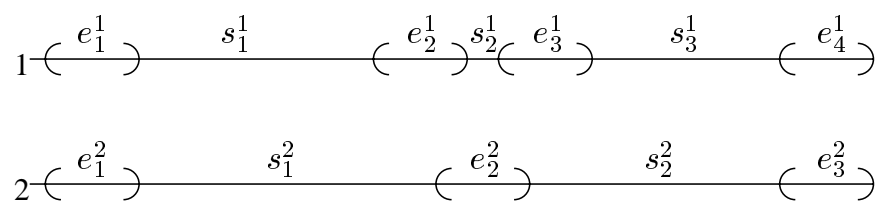

Figure 9: A computation $c_{2}$.

Since a minimal increase in $\left\langle C G S^{\stackrel{p b}{\rightarrow}}(c), \preceq\right\rangle$ does not necessarily correspond to advancing one process by one event, the algorithms in Section 5 cannot be adapted easily to detect Inst. Our algorithm for detecting Inst is based on Fromentin and Raynal's algorithm for detecting Properly in asynchronous systems $[9,10]$. The definition of Properly, generalized to an arbitrary ordering on events, is:

Properly: A computation $c$ satisfies Properly $\hookrightarrow \Phi$ iff there is a global state satisfying $\Phi$ and contained in every path of $\left\langle C G S^{\hookrightarrow}(c), \preceq\right\rangle$.

Theorem 7. Properly $\stackrel{d b}{\rightarrow}$ is equivalent to Inst.

Proof. It suffices to show that a global state $g$ is in $C G S^{\stackrel{p b}{\rightarrow}}(c)$ iff it is contained in every maximal path of $C G S \stackrel{d b}{\rightarrow}(c)$. The proof is based on Theorem SGS of [10], which states that a global state $g$ is contained in every maximal path of $\left\langle C G S^{\stackrel{h b}{\rightarrow}}(c), \preceq\right\rangle$ iff

$$
(\forall i, j: \mathcal{S}(g(i)) \stackrel{h b}{\rightarrow} \mathcal{T}(g(j)) \vee g(j)=\operatorname{last}(c(j))) \vee g(i)=\operatorname{first}(c(i))
$$

where first and last return the first and last element, respectively, of a sequence. A closely analogous proof shows that a global state $g$ is contained in every maximal path of $\left\langle C G S^{\stackrel{d b}{\rightarrow}}(c), \preceq\right\rangle$ iff $(\forall i, j$ : $\mathcal{S}(g(i)) \stackrel{d b}{\rightarrow} \mathcal{T}(g(j)))$, which by definition of $\stackrel{d b}{\rightarrow}$ is equivalent to

$$
(\forall i, j: i \neq j \Rightarrow \operatorname{upr}(\mathcal{S}(g(i)))<\operatorname{lwr}(\mathcal{T}(g(j)))) .
$$

The only significant difference involves the first and last local states of each process. Informally, the disjuncts $g(j)=$ last $(c(j))$ and $g(i)=f i r s t(c(i))$ are needed in Fromentin and Raynal's analysis because the global state $g_{i}$ containing the first local state of each process and the global state $g_{f}$ containing the last local state of each process both appear in every maximal path of $\langle C G S \stackrel{h b}{\longrightarrow}(c), \preceq\rangle$, even though the system might not pass through $g_{i}$ or $g_{f}$ in real-time, since the processes might start and finish at different times. This peculiarity does not arise when real-time timestamps are used, so (12) does not need disjuncts dealing specially with the first and last local states of each process. Expanding the definition of $C G S \stackrel{p b}{\rightarrow}(c)$ and simplifying yields (12). 
As an example of this equivalence, note that $\left\langle C G S \stackrel{p b}{\rightarrow}\left(c_{1}\right), \preceq\right\rangle$ in Figure 8 contains exactly the CGSs that are contained in every path of $\left\langle C G S \stackrel{d b}{\rightarrow}\left(c_{1}\right), \preceq\right\rangle$ in Figure 1 .

A straightforward adaptation of Fromentin and Raynal's algorithm for detecting Properly $\stackrel{h b}{\longrightarrow}$ yields an algorithm for detecting Inst. The heart of the algorithm is a procedure makeSC ("make Strongly Consistent") that loops until the heads of the queues form a SCGS or some queue is empty. Expanding the definition of consis $\stackrel{p b}{\rightarrow}(g)$ yields

$$
(\forall i, j: i \neq j \Rightarrow \operatorname{lwr}(\mathcal{T}(g(i)))>\operatorname{upr}(\mathcal{S}(g(j))))
$$

From this, one can show that, if the global state $\left(\lambda i \operatorname{head}\left(q_{i}\right)\right)$ is not strongly consistent, then in order to find a SCGS, some process $i$ such that $\operatorname{lwr}\left(\mathcal{T}\left(\right.\right.$ head $\left.\left.\left(q_{i}\right)\right)\right)$ is minimal must be advanced. This leads to the following pseudo-code for makeSC.

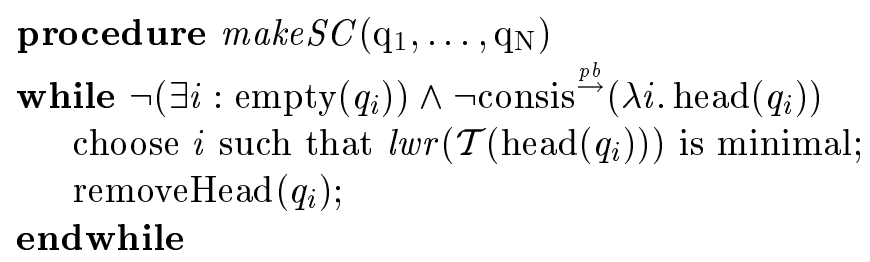

Our algorithm for detecting Inst appears in Figure 10, where head2 $(q)$ returns the second element of a queue $q$. Variable $a t S C$ is true when the heads of the queues form a SCGS. When a SCGS $g$ is found, if $g$ does not satisfy $\Phi$, then the algorithm starts searching for the next SCGS by advancing some process $j$ such that this advance yields a CGS (i.e., an element of $C G S \stackrel{d b}{\rightarrow}$ ).

We now describe some optimizations to the basic algorithm. The optimizations are very similar to those used in Section 5.1. To evaluate (13) efficiently, we modify the algorithm to maintain priority queues $p_{1}$ and $p_{2}$, whose contents are determined by the following invariants:

J1: For each process $i$ such that $q_{i}$ is non-empty, $p_{1}$ contains a record with key $\operatorname{lwr}\left(\mathcal{T}\left(\operatorname{head}\left(q_{i}\right)\right)\right)$ and satellite data $\langle i, p t r\rangle$, where $p t r$ is a pointer to the record with satellite data $i$ in $p_{2} . p_{1}$ contains no other records.

J2: For each process $i$ such that $q_{i}$ is non-empty, $p_{2}$ contains a record with key $u p r\left(\mathcal{S}\left(\operatorname{head}\left(q_{i}\right)\right)\right)$ and satellite data $i$. $p_{2}$ contains no other records.

Whenever the head of a queue changes, $p_{1}$ and $p_{2}$ are updated in $O(\log N)$ time to maintain these invariants. From $(13)$, one can show that consis $\stackrel{p b}{\rightarrow}\left(\lambda i \cdot \operatorname{head}\left(q_{i}\right)\right)$ is equivalent to

$$
\begin{aligned}
& \operatorname{key}\left(\operatorname{getMin}\left(p_{1}\right)\right)>\operatorname{key}\left(\operatorname{getMax}\left(p_{2}\right)\right) \\
& \vee\left(\pi_{1}\left(\operatorname{data}\left(\operatorname{getMin}\left(p_{1}\right)\right)\right)=\pi_{1}\left(\operatorname{data}\left(\operatorname{getMax}\left(p_{2}\right)\right)\right) \wedge \operatorname{countMax}\left(p_{2}\right)=1\right)
\end{aligned}
$$

where count $\operatorname{Max}(p)$ is the number of records containing the maximal value of the key in priority queue $p$, and data $(\langle k, d\rangle)=d$ and $\pi_{1}(\langle i, p t r\rangle)=i$. The second disjunct takes into account the possibility that $\operatorname{lwr}\left(\mathcal{T}\left(\operatorname{head}\left(q_{i}\right)\right)\right)<\operatorname{upr}\left(\mathcal{S}\left(\operatorname{head}\left(q_{i}\right)\right)\right)$ for some $i$. In this case, the first disjunct is 


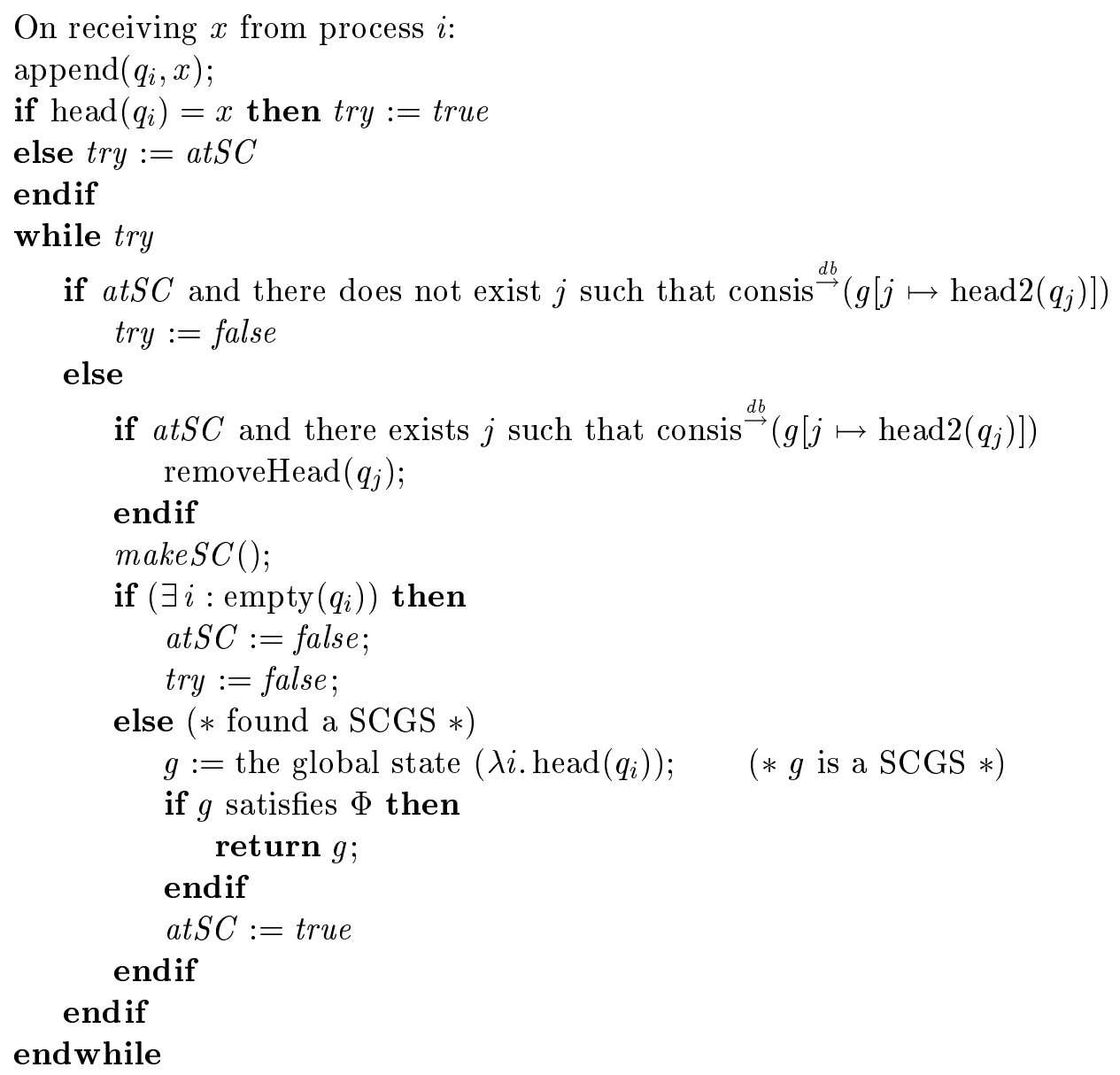

Figure 10: Algorithm for detecting Inst $\Phi$.

false (and the first conjunct of the second disjunct is true), but if there is no process $j$ different from $i$ and with $\operatorname{upr}\left(\mathcal{S}\left(\right.\right.$ head $\left.\left.\left(q_{j}\right)\right)\right)=\operatorname{upr}\left(\mathcal{S}\left(\operatorname{head}\left(q_{i}\right)\right)\right.$ ) (in which case the second conjunct of the second disjunct is also true), then the global state $\left(\lambda i \operatorname{head}\left(q_{i}\right)\right)$ is strongly consistent. In the pseudo-code for makeSC, consis $\stackrel{p b}{\rightarrow}\left(\lambda i\right.$. head $\left.\left(q_{i}\right)\right)$ can be replaced with (14), and line (†) can be replaced with $\langle k,\langle i, p t r\rangle\rangle:=\operatorname{extractMin}\left(p_{1}\right)$.

To efficiently evaluate the lines $(\ddagger)$ in Figure 10, observe that, if advancing some process yields a CGS (i.e., an element of $\left.C G S^{d b}\right)$, then advancing some process $j$ such that $l w r\left(\mathcal{S}\left(\operatorname{head} 2\left(q_{j}\right)\right)\right.$ ) is minimal yields a CGS; this follows from the definitions of $C G S \stackrel{d b}{\rightarrow}$ and $C G S \stackrel{p b}{\rightarrow}$. To efficiently find such a process $j$, we modify the algorithm to maintain a priority queue $p_{3}$ satisfying the invariant:

J3: For each process $i$ such that $q_{i}$ is non-empty, $p_{3}$ contains a record with key $\operatorname{lwr}\left(\mathcal{S}\left(\right.\right.$ head $\left.\left.2\left(q_{j}\right)\right)\right)$ and satellite data $i . p_{3}$ contains no other records.

Whenever a queue changes, $p_{3}$ is updated in $O(\log N)$ time to maintain this invariant. Thus, in the lines $(\ddagger)$ in Figure 10 , it suffices to take $j=\operatorname{head}\left(p_{3}\right)$. Testing whether $g\left[j \mapsto \operatorname{head} 2\left(q_{j}\right)\right]$ is 
consistent can be done in $O(\log N)$ time by temporarily updating all the priority queues as if process $j$ had been advanced and then using (10); note that the use of (10) requires that the algorithm maintain two additional priority queues, corresponding to invariants I1 and I2.

To evaluate $\left(\exists i: \operatorname{empty}\left(q_{i}\right)\right)$ efficiently, we modify the algorithm to maintain, as in Section 5.1, a natural number numEmpty, whose value is determined by the invariant: numEmpty = $\left|\left\{i \mid \operatorname{empty}\left(q_{i}\right)\right\}\right|$. In Figure 10 and in the code for makeSC, $\left(\exists i: \operatorname{empty}\left(q_{i}\right)\right)$ can be replaced with numEmpty $>0$.

We analyze the worst-case time complexity of the optimized algorithm by summing the times spent inside and outside of makeSC. Each iteration of the while loop in makeSC takes $O(\log N)$ time (because each operation on priority queues takes $O(\log N)$ time) and removes one local state. The computation contains $O(N E)$ local states, so the total time spent inside makeSC is $O((N \log N) E)$. The total time spent in the code outside makeSC is also $O((N \log N) E)$, since there are $O(N E)$ SCGSs (this is a corollary of Theorem 7), and each local state is considered at most once and at $O(\log N)$ cost. Thus, the worst-case time complexity of the algorithm is $O((N \log N) E)$.

\section{Sample Applications}

\subsection{Coherence Protocols}

Coherence of shared data is a central issue in many distributed systems, including distributed file systems, distributed shared memory, and distributed databases. A typical invariant maintained by a coherence protocol is:

cohrnt: if one machine has a copy of a data item in write mode, then no other machine has a valid copy of that data item.

As part of testing and debugging a coherence protocol, a monitor might be used to issue a warning if Poss $\stackrel{d b}{\rightarrow} \neg$ cohrnt is detected and report an error if Def $\stackrel{d b}{\rightarrow} \neg$ cohrnt is detected. A computationally cheaper but sometimes less informative alternative is to monitor only Inst $\neg$ cohrnt and report an error if it is detected.

A detection algorithm based on happened-before could be used instead, if the system can be modified to maintain vector clocks (or for some reason maintains them already). However, if the coherence protocol uses timers, then time acts as a hidden channel [2] (i.e., a means of communication other than messages), so detection based on happened-before might yield less accurate results. Passage of time can be used in coherence protocols to:

- obtain a lock, by broadcasting a request for a lock and, if no conflicting announcement is received within an appropriate time interval, granting oneself the lock.

- release a lock, by associating an expiration time with the lock; such a lock is called a lease [15]. When the lease expires, all processes know (without further communication) that the lock has been released. 
For example, both of these techniques are used in the resource allocation algorithm in [20, Section 5.1]. Detection based on happened-before is less appropriate for systems that use passage of time (instead of message transmission) for synchronization. For example, release of a lock by one process and acquisition of that lock by another process need not be related by happened-before, so Poss $\stackrel{h b}{\stackrel{h}{\rightarrow}} \neg$ cohrnt may be detected even when coherence was maintained and Poss $\stackrel{d b}{\rightarrow} \neg$ cohrnt would not be detected.

Clock-based monitoring is useful even for coherence protocols that provide weaker guarantees than cohrnt. For example, in the Sun Network File System (NFS) [31, Section 17.6.2], file information is cached. Timers are used to limit staleness: if the cached information is needed again after the timer expires, the client asks the server whether the cached information is still valid. Since this lock-free approach does not enforce the one-copy file-sharing semantics that is traditional in UNIX, it is useful to monitor the system to detect how often violations of one-copy semantics are seen by applications. For example, the detection algorithm for Inst can easily be adapted to count (instead of just detect) SCGSs satisfying the predicate: some process is reading cached information and some other cache contains a more recent version of that information. In NFS, the lifetime of cached data is typically tens of seconds, which is three orders of magnitude larger than typical clock synchronization error in a LAN, so this approach should detect most violations of one-copy semantics.

\subsection{Concurrency Control for Distributed Transactions}

Leases can also be used for concurrency control in distributed database systems, to reduce the number of messages needed to commit read-only transactions, as described in [23, Section 7]. The idea is that a read-only transaction acquires leases as it uses data objects. If the transaction completes before any of those leases expires, then the coordinator commits the transaction, without further communication. As part of testing and debugging such a system, one might use a monitor to detect violations of the invariant: when a transaction commits, it holds locks on all of the objects it uses. Since commit events and expirations of leases may be unrelated by happened-before, detection

based on Poss $\stackrel{h b}{\rightarrow}$ or Def $\stackrel{h b}{\rightarrow}$ may report violations even when no violation occurred and detection based on real-time clocks would not report a violation.

\section{References}

[1] Sridhar Alagar and S. Venkatesan. Techniques to tackle state explosion in global predicate detection. In Proc. International Conference on Parallel and Distributed Systems, pages 412417, December 1994.

[2] Özalp Babaoğlu and Keith Marzullo. Consistent global states of distributed systems: Fundamental concepts and mechanisms. In Sape Mullender, editor, Distributed Systems, chapter 5, pages 97-145. Addison Wesley, 2nd edition, 1993. 
[3] Özalp Babaoğlu and Michel Raynal. Specification and verification of dynamic properties in distributed computations. Journal of Parallel and Distributed Computing, 28(2):173-185, 1995.

[4] Bernadette Charron-Bost, Carole Delporte-Gallet, and Hugues Fauconnier. Local and temporal predicates in distributed systems. ACM Transactions on Programming Languages and Systems, 17(1):157-179, January 1995.

[5] Craig M. Chase and Vijay K. Garg. Detection of global predicates: Techniques and their limitations. Distributed Computing, 11(4):169-189, 1998.

[6] Robert Cooper and Keith Marzullo. Consistent detection of global predicates. In Proc. ACM/ONR Workshop on Parallel and Distributed Debugging, 1991. ACM SIGPLAN Notices 26(12):167-174, Dec. 1991.

[7] B. A. Davey and H. A. Priestley. Introduction to Lattices and Order. Cambridge University Press, 1990.

[8] Claire Diehl, Claude Jard, and Jean-Xavier Rampon. Reachability analysis on distributed executions. In J.-P. Jouannaud and M.-C. Gaudel, editors, TAPSOFT '93: Theory and Practice of Software Development, volume 668 of Lecture Notes in Computer Science, pages 629-643. Springer-Verlag, 1993.

[9] Eddy Fromentin and Michel Raynal. Characterizing and detecting the set of global states seen by all observers of a distributed computation. In Proc. IEEE 15th International Conference on Distributed Computing Systems (ICDCS), 1995.

[10] Eddy Fromentin and Michel Raynal. Shared global states in distributed computations. Journal of Computer and System Sciences, 55(3):522-528, 1997.

[11] Eddy Fromentin, Michel Raynal, Vijay K. Garg, and Alex Tomlinson. On-the-fly testing of regular patterns in distributed computations. In Proc. 23rd International Conference on Parallel Processing, volume 2, pages 73-76, August 1994.

[12] Vijay Garg, Craig Chase, Richard Kilgore, and J. Roger Mitchell. Efficient detection of channel predicates in distributed systems. Journal of Parallel and Distributed Computing, 45(2):134147, September 1997.

[13] Vijay K. Garg and Brian Waldecker. Detection of weak unstable predicates in distributed programs. IEEE Transactions on Parallel and Distributed Systems, 5(3):299-307, 1994.

[14] Vijay K. Garg and Brian Waldecker. Detection of strong unstable predicates in distributed programs. IEEE Transactions on Parallel and Distributed Systems, 7(12):1323-1333, 1996.

[15] C. Gray and D. Cheriton. Leases: An efficient fault-tolerant mechanism for distributed file system consistency. In Proc. 11th ACM Symposium on Operating System Principles, pages 202-210, 1989.

[16] Michel Hurfin, Masaaki Mizuno, Michel Raynal, and Mukesh Singhal. Efficient distributed detection of conjunctions of local predicates. IEEE Transactions on Software Engineering, 24(8):664-677, August 1998. 
[17] Michel Hurfin, Noël Plouzeau, and Michel Raynal. Detecting atomic sequences of predicates in distributed computations. In Proc. ACM/ONR Workshop on Parallel and Distributed Debugging, 1993.

[18] R. Jegou, R. Medina, and L. Nourine. Linear space algorithm for on-line detection of global predicates. In J. Desel, editor, Proc. Int'l Workshop on Structures in Concurrency Theory (STRICT '95). Springer, 1995.

[19] Leslie Lamport. Time, clocks, and the ordering of events in a distributed system. Communications of the ACM, 21(7):558-564, 1978.

[20] Leslie Lamport. Using time instead of timeout in fault-tolerant systems. ACM Transactions on Programming Languages and Systems, 6(2):256-280, April 1984.

[21] Leslie Lamport. The mutual exclusion problem: Part i-a theory of interprocess communication. Journal of the ACM, 33(2):313-326, 1986.

[22] Leslie Lamport. On interprocess communication: Part 1. Distributed Computing, 1:76-101, 1986.

[23] Barbara Liskov. Practical uses of synchronized clocks in distributed systems. In Proc. Tenth ACM Symposium on Principles of Distributed Computing, pages 1-9. ACM Press, August 1991.

[24] Keith Marzullo and Gil Neiger. Detection of global state predicates. In Proc. 5th Int'l. Workshop on Distributed Algorithms (WDAG), volume 579 of Lecture Notes in Computer Science, pages 254-272. Springer, 1991.

[25] Jean Mayo and Phil Kearns. Global predicates in rough real time. In Proc. 7th IEEE Symposium on Parallel and Distributed Processing (SPDP), pages 17-24, 1995.

[26] David L. Mills. Internet time synchronization: the Network Time Protocol. IEEE Trans. Communications, 39(10):1482-1493, October 1991.

[27] David L. Mills. Network time protocol (version 3) specification, implementation and analysis. DARPA Network Working Group Report RFC-1305, University of Delaware, March 1992.

[28] David L. Mills. Improved algorithms for synchronizing computer network clocks. IEEE/ACM Transactions on Networking, 3(3):245-254, June 1995.

[29] OSF. Introduction to OSF DCE. Prentice-Hall, 1992.

[30] Reinhard Schwarz and Friedemann Mattern. Detecting causal relationships in distributed computations: In search of the holy grail. Distributed Computing, 7(3):149-174, 1994.

[31] Abraham Silberschatz and Peter B. Galvin. Operating System Concepts. Addison Wesley, 4th edition, 1994.

[32] Scott D. Stoller. Detecting global predicates in distributed systems with clocks. In Marios Mavronikolas, editor, Proc. 11th International Workshop on Distributed Algorithms (WDAG '97), volume 1320 of Lecture Notes in Computer Science, pages 185-199. Springer-Verlag, 1997. 
[33] Scott D. Stoller and Yanhong A. Liu. Efficient symbolic detection of global properties in distributed systems. In Alan J. Hu and Moshe Y. Vardi, editors, Proc. 10th Int'l. Conference on Computer-Aided Verification (CAV), volume 1427 of Lecture Notes in Computer Science, pages 357-368. Springer-Verlag, June 1998.

[34] Scott D. Stoller and Fred B. Schneider. Faster possibility detection by combining two approaches. In Jean-Michel Hélary and Michel Raynal, editors, Proc. 9th International Workshop on Distributed Algorithms (WDAG '95), volume 972 of Lecture Notes in Computer Science, pages 318-332. Springer-Verlag, September 1995.

[35] Paulo Veríssimo. Real-time communication. In Sape Mullender, editor, Distributed Systems, chapter 17, pages 447-490. Addison Wesley, 2nd edition, 1993. 